\title{
Positively Invariant Subset for Non-Densely Defined Cauchy Problems
}

\author{
Pierre Magal(a) and Ousmane Seydi ${ }^{(\mathrm{b})}$ \\ (a) Univ. Bordeaux, IMB, UMR 5251, F-33400 Talence, France \\ CNRS, IMB, UMR 5251, F-33400 Talence, France. \\ (b) Département Tronc Commun,École Polytechnique de Thiès, Sénégal
}

November 13, 2019

\begin{abstract}
Under weak Hille-Yosida conditions and using a generalized notion of sub tangential condition, we prove the positive invariance of a closed subset by the semiflow generated by a semi-linear non densely defined Cauchy problem. A simple remark shows that the sufficient condition for the positivity of the semiflow implies our sub tangentiality condition. But the sub tangential condition applies to a much larger class of closed positively invariant subset. Our results can be applied to hyperbolic and parabolic partial differential equations as well as functional differential equations. As an illustration we apply our results to an age-structured equation in $L^{p}$ spaces which is only defined on a closed subset of $L^{p}$.
\end{abstract}

Key words. Semilinear differential equations, non-dense domain, integrated semigroup, positively invariant subset, age structured population dynamics models.

AMS Subject Classification. 37N25, 92D25, 92D30

\section{Introduction}

In this article we consider an abstract semi-linear Cauchy problem

$$
\frac{d u(t)}{d t}=A u(t)+F(t, u(t)), \text { for } t \geq 0, \text { with } u(0)=u_{0} \in \overline{D(A)},
$$

where $A: D(A) \subset X \rightarrow X$ is a linear operator on a Banach space $X$, and $F:[0, \infty) \times \overline{D(A)} \rightarrow X$ is continuous. We assume that the map $x \rightarrow F(t, x)$ is Lipschitz on the bounded sets of $\overline{D(A)}$ uniformly with respect to $t$ in a bounded interval of $[0, \infty)$. We point out that $D(A)$ is not necessarily dense in $X$ and $A$ is not necessarily a Hille-Yosida operator. 
The invariance of subset for differential equation has a long history which starts with the seminal paper of the Japanese mathematician Nagumo [13] in 1942. The result for ordinary differential equations was rediscovered later on by Brezis [4] and Hartman [6] and was further extended to ordinary differential equation in ordered Banach spaces by Walter [19] and Redheffer and Walter [15]. Several extensions to partial differential equations were proposed later on by Redheffer and Walter [16] and Martin [11] for parabolic equations, etc. Martin and Smith [12] further investigated comparison/differential inequalities and invariant sets for abstract functional differential equations and reactiondiffusion systems that have time delays in the nonlinear reaction terms, and their developed results have had many applications. We refer to the book of Pavel and Motreanu [14] for an extensive study of densely defined semi-linear Cauchy problem. In [14] the authors studied the positive invariance for general closed subset subjected to tangency condition. They also conidered positive invariance of time dependent closed subset and extended their results to semilinear differential inclusion problems. The case of closed convex subset for nondensely defined Cauchy problems with a Hille-Yosida linear operator perturbed by Lipschitz continuous non linear map has been studied by Thieme [17]. The goal of this article is to extend the results of Thieme [17] from the Hille-Yosida case to the non Hille-Yosida case. It is worth noting that the non Hille-Yosida case induces several difficulties due to the problem of non uniform boundedness of $\lambda(\lambda-A)^{-1}$ whenever $\lambda$ becomes. To overcome these difficulties we use a somewhat different approach compared to Thieme [17] combined with some generalization of the estimates on the integrated semigroup from the space of continuous functions to the space of regulated functions. Thank to our weak Hille-Yosida condition on the linear operator $A$ in (1.1) (see Assumption 4.4) combined together with our generalized sub tangential condition (see Assumptions (2.1) and (2.4)) we can be applied our result to hyperbolic and parabolic partial differential equations in $L^{p}$ instead of $L^{1}$.

The paper is organized as follows. In sections 2 and 3 we recall some basic results about non densely defined Cauchy problems. In section 4 , we investigate the positive invariance of a closed subset. In section 5, we apply our result to an age-structured equation in $L^{p}$ spaces which only defined in a closed subset of $L^{p}$ and show that it generates a semiflow.

\section{Preliminary results}

Let $A: D(A) \subset X \rightarrow X$ be a linear operator. In the following we use the following notations

$$
X_{0}:=\overline{D(A)}
$$

and $A_{0}: D\left(A_{0}\right) \subset X_{0} \rightarrow X_{0}$ the part of $A$ in $X_{0}$ that is

$$
A_{0} x=A x, \quad \forall x \in D\left(A_{0}\right),
$$

and

$$
D\left(A_{0}\right)=\left\{x \in D(A): A x \in X_{0}\right\} .
$$


Assumption 2.1 We assume that

(i) There exist two constants $\omega_{A} \in \mathbb{R}$ and $M_{A} \geq 1$, such that $\left(\omega_{A},+\infty\right) \subset$ $\rho(A)$ and

$$
\left\|(\lambda I-A)^{-n}\right\|_{\mathcal{L}\left(X_{0}\right)} \leq M_{A}\left(\lambda-\omega_{A}\right)^{-n}, \forall \lambda>\omega_{A}, \forall n \geq 1 .
$$

(ii) $\lim _{\lambda \rightarrow+\infty}(\lambda I-A)^{-1} x=0, \forall x \in X$.

It is important to note that Assumption 2.1 does not say that $A$ is a HilleYosida linear operator since the operator norm in Assumption 2.1-(i) is taken into $X_{0} \subseteq X$ (where the inclusion can be strict) instead of $X$. Further, it follows from [10] that $\rho(A)=\rho\left(A_{0}\right)$. Therefore by Assumption 2.1, $\left(A_{0}, D\left(A_{0}\right)\right)$ is a Hille-Yosida linear operator of type $\left(\omega_{A}, M_{A}\right)$ and generates a strongly continuous semigroup $\left\{T_{A_{0}}(t)\right\}_{t \geq 0} \subset \mathcal{L}\left(X_{0}\right)$ with

$$
\left\|T_{A_{0}}(t)\right\|_{\mathcal{L}\left(X_{0}\right)} \leq M_{A} e^{\omega_{A} t}, \quad \forall t \geq 0 .
$$

As a consequence

$$
\lim _{\lambda \rightarrow+\infty} \lambda(\lambda I-A)^{-1} x=x
$$

only for $x \in X_{0}$. It is important to note that the above limit does not exist in general whenever $x$ belongs to $X$.

We summarize the above discussions as follows.

Lemma 2.2 Assumption 2.1 is satisfied if and only if there exist two constants, $M_{A} \geq 1$ and $\omega_{A} \in \mathbb{R}$, such that $\left(\omega_{A},+\infty\right) \subset \rho(A)$ and $A_{0}$ is the infinitesimal generator of a $C_{0}$-semigroup $\left\{T_{A_{0}}(t)\right\}_{t \geq 0}$ on $X_{0}$ which satisfies $\left\|T_{A_{0}}(t)\right\|_{\mathcal{L}\left(X_{0}\right)} \leq$ $M_{A} e^{\omega_{A} t}, \forall t \geq 0$.

Next, we consider the non homogeneous Cauchy problem

$$
v^{\prime}(t)=A v(t)+f(t), \quad t \geq 0 \quad \text { and } \quad v(0)=v_{0} \in X_{0},
$$

with $f \in L_{\text {loc }}^{1}(\mathbb{R}, X)$.

The integrated semi-group is one of the major tools to investigate nonhomogeneous Cauchy problems. This notion was first introduced by Ardent $[1,2]$. We refer to the books Arendt et al. [3] whenever $A$ an Hille-Yosida operator. We refer to Magal and Ruan $[8,10]$ and Thieme [18] for an integrated semi-group theory whenever $A$ is not Hille-Yosida operator. We also refer to the book of Magal and Ruan [10] for more references and results on this topic.

Definition 2.3 Let Assumption 2.1 be satisfied. Then $\left\{S_{A}(t)\right\}_{t \geq 0} \in \mathcal{L}(X)$ the integrated semigroup generated by $A$ is a strongly continuous family of bounded linear operators on $X$, which is defined by

$$
S_{A}(t) x=\left(\lambda I-A_{0}\right) \int_{0}^{t} T_{A_{0}}(l)(\lambda I-A)^{-1} x d l, \forall t \geq 0 .
$$


In order to obtain existence and uniqueness of solutions for (2.1) whenever $f$ is a continuous map, we will require the following assumption.

Assumption 2.4 Assume that for any $\tau>0$ and $f \in C([0, \tau], X)$ there exists $v_{f} \in C\left([0, \tau], X_{0}\right)$ an integrated (mild) solution of

$$
\frac{d v_{f}(t)}{d t}=A v_{f}(t)+f(t), \text { for } t \geq 0 \text { and } v_{f}(0)=0,
$$

that is to say that

$$
\int_{0}^{t} v_{f}(r) d r \in D(A), \forall t \geq 0
$$

and

$$
v_{f}(t)=A \int_{0}^{t} v_{f}(r) d r+\int_{0}^{t} f(r) d r, \forall t \geq 0 .
$$

Moreover we assume that there exists a non decreasing map $\delta:[0,+\infty) \rightarrow$ $[0,+\infty)$ such that

$$
\left\|v_{f}(t)\right\| \leq \delta(t) \sup _{s \in[0, t]}\|f(s)\|, \forall t \geq 0,
$$

with $\delta(t) \rightarrow 0$ as $t \rightarrow 0^{+}$.

Remark 2.5 Note that Assumption 2.4 is equivalent (see [9]) to the assumption that there exists a non-decreasing map $\delta:[0,+\infty) \rightarrow[0,+\infty)$ such that for each $\tau>0$ and each $f \in C([0, \tau], X)$ the map $t \rightarrow\left(S_{A} * f\right)(t)$ is differentiable in $[0, \tau]$ with

$$
\left\|\left(S_{A} \diamond f\right)(t)\right\| \leq \delta(t) \sup _{s \in[0, t]}\|f(s)\|, \forall t \in[0, \tau],
$$

where $\left(S_{A} * f\right)(t)$ and $\left(S_{A} \diamond f\right)(t)$ will be defined below in Theorem 2.7 and equation (2.3).

Remark 2.6 It is important to point out the fact Assumption 2.4 is also equivalent to saying that $\left\{S_{A}(t)\right\}_{t \geq 0} \subset \mathcal{L}\left(X, X_{0}\right)$ is of bounded semi-variation on $[0, t]$ for any $t>0$ that is to say that

$$
V^{\infty}\left(S_{A}, 0, t\right):=\sup \left\{\left\|\sum_{i=0}^{n-1}\left[S_{A}\left(t_{j+1}\right)-S_{A}\left(t_{j}\right)\right] x_{j}\right\|\right\}<+\infty
$$

where the supremum is taken over all partitions $0=t_{0}<\cdots<t_{n}=t$ of $[0, t]$ and all elements $x_{1}, \ldots, x_{n} \in X$ with $\left\|x_{j}\right\| \leq 1$, for $j=1,2, \ldots, n$. Moreover the non-decreasing map $\delta:[0,+\infty) \rightarrow[0,+\infty)$ in Assumption 2.4 is defined by

$$
\delta(t):=\sup _{s \in[0, t]} V^{\infty}\left(S_{A}, 0, s\right), \forall t \geq 0 .
$$

The following result is proved in [9, Theorem 2.9]. 
Theorem 2.7 Let Assumptions 2.1 and 2.4 be satisfied. Then for each $\tau>0$ and each $f \in C([0, \tau], X)$ the map

$$
t \rightarrow\left(S_{A} * f\right)(t):=\int_{0}^{t} S_{A}(t-s) f(s) d s
$$

is continuously differentiable, $\left(S_{A} * f\right)(t) \in D(A), \forall t \in[0, \tau]$, and if we set $u(t)=\frac{d}{d t}\left(S_{A} * f\right)(t)$, then

$$
u(t)=A \int_{0}^{t} u(s) d s+\int_{0}^{t} f(s) d s, \forall t \in[0, \tau] .
$$

Moreover we have

$$
\|u(t)\| \leq \delta(t) \sup _{s \in[0, t]}\|f(s)\|, \forall t \in[0, \tau] .
$$

Furthermore, for each $\lambda \in(\omega,+\infty)$ we have for each $t \in[0, \tau]$ that

$$
(\lambda I-A)^{-1} \frac{d}{d t}\left(S_{A} * f\right)(t)=\int_{0}^{t} T_{A_{0}}(t-s)(\lambda I-A)^{-1} f(s) d s .
$$

From now on we will use the following notation

$$
\left(S_{A} \diamond f\right)(t):=\frac{d}{d t}\left(S_{A} * f\right)(t) .
$$

From $(2.2)$ and using the fact that $\left(S_{A} \diamond f\right)(t) \in X_{0}$, we deduce the approximation formula

$$
\left(S_{A} \diamond f\right)(t)=\lim _{\lambda \rightarrow+\infty} \int_{0}^{t} T_{A_{0}}(t-s) \lambda(\lambda I-A)^{-1} f(s) d s .
$$

A consequence of the approximation formula is the following

$$
\left(S_{A} \diamond f\right)(t+s)=T_{A_{0}}(s)\left(S_{A} \diamond f\right)(t)+\left(S_{A} \diamond f(t+.)\right)(s), \forall t, s \geq 0 .
$$

The following result is proved by Magal and Ruan [8, Theorem 3.1], which will be constantly used and applied to the operator $A-\gamma B$ in sections 4 and 5 .

\section{Theorem 2.8 (Bounded Linear Perturbation)}

Let Assumptions 2.1 and 2.4 be satisfied. Assume $L \in \mathcal{L}\left(X_{0}, X\right)$ is a bounded linear operator. Then $A+L: D(A) \subset X \rightarrow X$ satisfies the conditions in Assumptions 2.1 and 2.4. More precisely, if we fix $\tau_{L}>0$ such that

$$
\delta\left(\tau_{L}\right)\|L\|_{\mathcal{L}\left(X_{0}, X\right)}<1,
$$

and if we denote by $\left\{S_{A+L}(t)\right\}_{t \geq 0}$ the integrated semigroup generated by $A+L$, then for any $f \in C\left(\left[0, \tau_{L}\right], X\right)$, we have

$$
\left\|\frac{d}{d t}\left(S_{A+L} * f\right)\right\| \leq \frac{\delta(t)}{1-\delta\left(\tau_{L}\right)\|L\|_{\mathcal{L}\left(X_{0}, X\right)}} \sup _{s \in[0, t]}\|f(s)\|, \forall t \in\left[0, \tau_{L}\right] .
$$


The following result is proved in [9, Lemma 2.13].

Lemma 2.9 Let Assumptions 2.1 and 2.4 be satisfied. Then

$$
\lim _{\lambda \rightarrow+\infty}\left\|(\lambda I-A)^{-1}\right\|_{\mathcal{L}(X)}=0 .
$$

It follows that if $B \in \mathcal{L}\left(X_{0}, X\right)$, then for all $\lambda>0$ large enough the linear operator $\lambda I-A-B$ is invertible and its inverse can be written as follows

$$
(\lambda I-A-B)^{-1}=(\lambda I-A)^{-1}\left[I-B(\lambda I-A)^{-1}\right]^{-1} .
$$

\section{Existence and Uniqueness of a Maximal Semi- flow}

Consider now the non-autonomous semi-linear Cauchy problem

$$
U(t, s) x=x+A \int_{s}^{t} U(l, s) x d l+\int_{s}^{t} F(l, U(l, s) x) d l, \quad t \geq s \geq 0,
$$

and the following problem

$$
U(t, s) x=T_{A_{0}}(t-s) x+\frac{d}{d t}\left(S_{A} * F(.+s, U(.+s, s) x)(t-s), t \geq s \geq 0 .\right.
$$

We will make the following assumption.

Assumption 3.1 Assume that $F:[0,+\infty) \times \overline{D(A)} \rightarrow X$ is a continuous map such that for each $\tau_{0}>0$ and each $\xi>0$, there exists $K\left(\tau_{0}, \xi\right)>0$ such that

$$
\|F(t, x)-F(t, y)\| \leq K\left(\tau_{0}, \xi\right)\|x-y\|
$$

whenever $t \in\left[0, \tau_{0}\right], y, x \in X_{0}$, and $\|x\| \leq \xi,\|y\| \leq \xi$.

In the following definition $\tau$ is the blow-up time of maximal solutions of (3.1).

Definition 3.2 (Non autonomous maximal semiflow)

Consider two maps $\tau:[0,+\infty) \times X_{0} \rightarrow(0,+\infty]$ and $U: D_{\tau} \rightarrow X_{0}$, where

$$
D_{\tau}=\left\{(t, s, x) \in[0,+\infty)^{2} \times X_{0}: s \leq t<s+\tau(s, x)\right\} .
$$

We say that $U$ is a maximal non-autonomous semiflow on $X_{0}$ if $U$ satisfies the following properties

(i) $\tau(r, U(r, s) x)+r=\tau(s, x)+s, \forall s \geq 0, \forall x \in X_{0}, \forall r \in[s, s+\tau(s, x))$.

(ii) $U(s, s) x=x, \forall s \geq 0, \forall x \in X_{0}$.

(iii) $U(t, r) U(r, s) x=U(t, s) x, \forall s \geq 0, \forall x \in X_{0}, \forall t, r \in[s, s+\tau(s, x))$ with $t \geq r$. 
(iv) If $\tau(s, x)<+\infty$, then

$$
\lim _{t \rightarrow(s+\tau(s, x))^{-}}\|U(t, s) x\|=+\infty .
$$

Set

$$
D=\left\{(t, s, x) \in[0,+\infty)^{2} \times X_{0}: t \geq s\right\} .
$$

The following theorem is the main result in this section, which was proved in [8, Theorem 5.2].

Theorem 3.3 Let Assumptions 2.1, 2.4 and 3.1 be satisfied. Then there exists a map $\tau:[0,+\infty) \times X_{0} \rightarrow(0,+\infty]$ and a maximal non-autonomous semiflow $U: D_{\tau} \rightarrow X_{0}$, such that for each $x \in X_{0}$ and each $s \geq 0, U(., s) x \in$ $C\left([s, s+\tau(s, x)), X_{0}\right)$ is a unique maximal solution of (3.1) (or equivalently a unique maximal solution of (3.2)). Moreover, $D_{\tau}$ is open in $D$ and the map $(t, s, x) \rightarrow U(t, s) x$ is continuous from $D_{\tau}$ into $X_{0}$.

\section{Positive invariance of a closed subset}

In this section we will study the positive invariance of a closed subset by imposing the so called sub-tangential condition. Our results extend those in $[14,17]$ since we focus on the study of non densely defined non Hille-Yosida semilinear Cauchy problems. We start with some lemmas that will be useful in the subsequent discussions.

Lemma 4.1 Let Assumptions 2.1 and 2.4 be satisfied. Let $0 \leq a<b$ and $x \in X$ be given and define

$$
f(t):=x \mathbb{1}_{[a, b]}(t), \forall t \geq 0 .
$$

Then $t \rightarrow\left(S_{A} * f\right)(t)$ is differentiable in $[0,+\infty)$ and

$$
\left(S_{A} \diamond f\right)(t)=\frac{d}{d t}\left(S_{A} * f\right)(t)=S_{A}\left((t-a)^{+}\right) x-S_{A}\left((t-b)^{+}\right) x, \forall t \geq 0,
$$

where $\sigma^{+}:=\max (0, \sigma), \forall \sigma \in \mathbb{R}$.

Proof. We observe that

$$
\left(S_{A} * f\right)(t)=\left\{\begin{array}{lll}
\int_{a}^{t} S_{A}(t-s) x d s & \text { if } & t \in[a, b], \\
\int_{a}^{b} S_{A}(t-s) x d s & \text { if } & t \geq b, \\
0 & \text { if } & 0 \leq t \leq a,
\end{array}\right.
$$

which is equivalent to

$$
\left(S_{A} * f\right)(t)=\left\{\begin{array}{lll}
\int_{0}^{t-a} S_{A}(s) x d s & \text { if } & t \in[a, b] \\
\int_{t-b}^{t-a} S_{A}(s) x d s & \text { if } & t \geq b \\
0 & \text { if } & 0 \leq t \leq a
\end{array}\right.
$$


Then the formula follows by computing the time derivative.

By using similar arguments in the proof of Lemma 4.1 one can easily obtain the following results.

Lemma 4.2 Let Assumptions 2.1 and 2.4 be satisfied. Let $0 \leq a<b$ be given. Let $a=t_{0}<\cdots<t_{n}=b$ be a partition of $[a, b]$. Let $f:[a, b] \rightarrow X$ be the step function defined by

$$
f(t):=\sum_{i=0}^{n-1} x_{i} \mathbb{1}_{\left[t_{i}, t_{i+1}\right)}(t), \forall t \in[a, b) \text { and } f(b)=f\left(t_{n-1}\right)=x_{n-1} .
$$

Then $t \rightarrow\left(S_{A} * f(a+\cdot)\right)(t-a)$ is differentiable in $[a, b]$ and for any $t \in$ $\left[t_{k}, t_{k+1}\right], k=0, \ldots, n-1$ one has

$$
\left(S_{A} \diamond f(a+\cdot)\right)(t-a)=\sum_{i=0}^{k-1}\left[S_{A}\left(t-t_{i}\right)-S_{A}\left(t-t_{i+1}\right)\right] x_{i}+S_{A}\left(t-t_{k}\right) x_{k} .
$$

Recall that $f:[a, b] \rightarrow X$ is a regulated function if the limit from the right side $\lim _{s \rightarrow t^{+}} f(s)$ exists for each $t \in[a, b)$, and the limit from the left side $\lim _{s \rightarrow t^{-}} f(s)$ exists for each $t \in(a, b]$. For each $b>a \geq 0$, we assume $\operatorname{Reg}([a, b], X)$ denotes the space of regulated functions from $[a, b]$ to $X$, and we also denote by $\operatorname{Step}([a, b], X)$ the space of step functions from $[a, b]$ to $X$.

The following lemma extend the property described in Assumption 2.4 for the space of continuous functions to the space of regulated functions.

Lemma 4.3 Let Assumptions 2.1 and 2.4 be satisfied and let $0 \leq a<b$ be given. Then for any $f \in \operatorname{Reg}([a, b], X)$ we have

$$
\left\|\left(S_{A} \diamond f(a+\cdot)\right)(t-a)\right\| \leq \delta(t-a) \sup _{s \in[a, t]}\|f(s)\|, \forall t \in[a, b] .
$$

Proof. Since $\operatorname{Step}([a, b], X)$ is dense in $\operatorname{Reg}([a, b], X)$ for the topology of uniform convergence (see Dieudonne [5, p.139]), it is sufficient to prove the result for $f \in \operatorname{Step}([a, b], X)$ and apply the linear extension theorem to the bounded linear operator

$$
f \in \operatorname{Step}([a, b], X) \mapsto\left(S_{A} \diamond f\right)(\cdot) .
$$

Let $f \in \operatorname{Step}([a, b], X)$ be a non zero step function given by

$$
f(t):=\sum_{i=0}^{n-1} x_{i} \mathbb{1}_{\left[t_{i}, t_{i+1}\right)}(t), \forall t \in[a, b), \quad \text { and } f(b)=f\left(t_{n-1}\right)=x_{n-1}
$$

with $a=t_{0}<t_{1}<\cdots<t_{n}=b$. Let $t \in[a, b]$ be given and fixed. Then there exists $k \in\{0, \ldots, n-1\}$ such that $t \in\left[t_{k}, t_{k+1}\right]$. Hence by Lemma 4.2 we have

$$
\begin{aligned}
\left(S_{A} \diamond f(a+\cdot)\right)(t-a) & =\sum_{i=0}^{k-1}\left[S_{A}\left(t-t_{i}\right)-S_{A}\left(t-t_{i+1}\right)\right] x_{i}+S_{A}\left(t-t_{k}\right) x_{k} \\
& =\sum_{i=0}^{k} S_{A}\left(t-t_{k-i}\right) x_{k-i}-\sum_{i=1}^{k} S_{A}\left(t-t_{k-i+1}\right) x_{k-i}
\end{aligned}
$$


Setting

$$
\bar{t}_{i}=t-t_{k-i+1}, i=1, \ldots, k \text { and } \bar{t}_{0}:=0
$$

and

$$
\bar{x}_{i}:=\frac{x_{k-i}}{\alpha}, i=0, \ldots, k
$$

with $\alpha:=\max _{i=1, \ldots, k}\left\|x_{i}\right\|>0$. Then we obtain

$$
\left(S_{A} \diamond f(a+\cdot)\right)(t-a)=\alpha \sum_{i=0}^{k}\left[S_{A}\left(\bar{t}_{i+1}\right)-S_{A}\left(\bar{t}_{i}\right)\right] \bar{x}_{i} .
$$

Since $0=\bar{t}_{0}<\cdots<\bar{t}_{k+1}=t-a$ and $\left\|\bar{x}_{i}\right\| \leq 1$ for all $i=1, \ldots, k$, it follows from Remark 2.6 that

$$
\left\|\left(S_{A} \diamond f(a+\cdot)\right)(t-a)\right\| \leq \alpha V^{\infty}\left(S_{A}, 0, t-a\right) \leq \alpha \delta(t-a)
$$

and the result follows by observing that

$$
\alpha:=\max _{i=1, \ldots, k}\left\|x_{i}\right\|=\sup _{s \in[a, t]}\|f(s)\| .
$$

In order to prove the invariance property of a closed subset $C_{0} \subset X_{0}$ we need to make the following assumption.

Assumption 4.4 (Sub-Tangential Condition) Let $C_{0}$ be a closed subset of $X_{0}$. We assume that there exists a bounded linear operator $B: X_{0} \rightarrow X$ such that for each $\xi>0$ and each $\sigma>0$ there exists $\gamma=\gamma(\xi, \sigma)>0$ such that

$$
\lim _{h \rightarrow 0^{+}} \frac{1}{h} d\left(T_{(A-\gamma B)_{0}}(h) x+S_{A-\gamma B}(h)[F(t, x)+\gamma B x], C_{0}\right)=0,
$$

whenever $x \in C_{0}$ with $\|x\| \leq \xi$ and $t \in[0, \sigma]$. Here the map $x \rightarrow d\left(x, C_{0}\right)$ is the Hausdorff semi-distance which is defined as

$$
d\left(x, C_{0}\right):=\inf _{y \in C_{0}}\|x-y\| .
$$

Remark 4.5 Recall that the usual assumption for the non negativity of the mild solutions of (1.1) is covered by Assumption 4.4. In fact $X_{0+}$ is positively invariant with respect to semiflow generated by (1.1) if for each $\xi>0$ and each $\sigma>0$ there exists $\gamma=\gamma(\xi, \sigma)>0$ such that

$$
T_{(A-\gamma B)_{0}}(h) x+S_{A-\gamma B}(h)[F(t, x)+\gamma B x] \in X_{0+}
$$

whenever $x \in X_{0+}$ with $\|x\| \leq \xi$ and $t \in[0, \sigma]$.

The main result of this article is the following theorem.

Theorem 4.6 (Positive invariant Subset) Let Assumptions 2.1, 2.4, 3.1 and 4.4 be satisfied. Then for each $x \in C_{0}$ and each $s \geq 0$, we have

$$
U(t, s) x \in C_{0}, \forall t \in[s, s+\tau(s, x)) .
$$


The rest of this section is devoted to the proof of Theorem 4.6. We fix the initial condition $x_{0} \in C_{0}$ and $s=0$. Set $\rho:=2\left(\left\|x_{0}\right\|+1\right)$ and define

$$
F_{\gamma}(t, x):=F(t, x)+\gamma B x, \forall(t, x) \in[0,+\infty) \times X_{0} .
$$

Let $\Lambda:=\Lambda(\rho)>0$ be the constant such that

$$
\left\|F_{\gamma}(t, x)-F_{\gamma}(t, y)\right\| \leq \Lambda\|x-y\|, \forall t \in[0, \rho], \forall x, y \in B(0, \rho) .
$$

Therefore by setting

$$
\Gamma:=2 \Lambda \rho+\sup _{t \in[0, \rho]}\left\|F_{\gamma}\left(t, x_{0}\right)\right\|,
$$

we obtain

$$
\left\|F_{\gamma}(t, x)\right\| \leq \Gamma, \forall t \in[0, \rho], \forall x \in B(0, \rho) .
$$

Let $\gamma:=\gamma(\rho)>0$ be a constant such that

$$
\lim _{h \rightarrow 0^{+}} \frac{1}{h} d\left(T_{(A-\gamma B)_{0}}(h) x+S_{A-\gamma B}(h) F_{\gamma}(t, x), C_{0}\right)=0,
$$

whenever $x \in C_{0},\|x\| \leq \rho$ and $t \in[0, \rho]$.

Then by Theorem 2.8, $A-\gamma B: D(A) \subset X \rightarrow X$ satisfies Assumptions 2.1 and 2.4. Hence combining Theorem 2.8 and Lemma 4.3 we know that if we fix $\tau_{\gamma}>0$ such that

$$
\gamma \delta\left(\tau_{\gamma}\right)\|B\|_{\mathcal{L}\left(X_{0}, X\right)}<1,
$$

then there exists a non decreasing map $\delta_{\gamma}:[0,+\infty) \rightarrow[0,+\infty)$ with

$$
\lim _{t \rightarrow 0^{+}} \delta_{\gamma}(t)=0
$$

such that for each $f \in \operatorname{Reg}([a, b], X), 0 \leq a<b \leq \tau_{\gamma}$

$$
\left\|\left(S_{A-\gamma B} \diamond f(a+\cdot)\right)(t-a)\right\| \leq \delta_{\gamma}(t-a) \sup _{s \in[a, t]}\|f(s)\|, \forall t \in[a, b] .
$$

To shorten the notations we set

$$
\omega_{\gamma}:=\omega_{A-\gamma B} \text { and } M_{\gamma}:=M_{A-\gamma B} .
$$

Let $\tau \in\left(0, \min \left(\tau(0, x), \tau_{\gamma}, \rho\right)\right)$ be small enough to satisfy

$$
\Gamma \delta_{\gamma}(h)+M_{\gamma} e^{\omega_{\gamma}^{+} h} h+\left\|T_{(A-\gamma B)_{0}}(h) x_{0}\right\| \leq \rho, \forall h \in[0, \tau]
$$

with

$$
\omega_{\gamma}^{+}=\max \left(0, \omega_{\gamma}\right),
$$

and

$$
0<\Lambda \delta_{\gamma}(\tau)<1,
$$

where $\Lambda$ has been defined as an upper bound for the Lipschitz norm of $F_{\gamma}$ on $B(0, \rho) \cap C_{0}$ in $(4.1)$. 
Construction of the knots : Let $\varepsilon \in(0,1)$ be fixed. We define by induction a sequence $\left(l_{k}, y_{k}\right) \in[0, \tau] \times C_{0}$ where the index $k \in \mathbb{N}$ is a non-negative integer possibly unbounded. For $k=0$ we start with

$$
l_{0}=0 \text { and } y_{0}=x_{0} \in C_{0} .
$$

In order to compute the next increment, we define for each integer $k \geq 0$

$$
\begin{aligned}
I_{k}=\left\{\eta \in\left(0, \varepsilon^{*}\right): \quad\right. & \left\|F_{\gamma}(l, y)-F_{\gamma}\left(l_{k}, y_{k}\right)\right\| \leq \varepsilon, \forall\left|l-l_{k}\right| \leq \eta, \forall y \in B\left(y_{k}, \eta\right) \cap C_{0}, \\
& \frac{1}{\eta} d\left(T_{(A-\gamma B)_{0}}(\eta) y_{k}+S_{A-\gamma B}(\eta) F_{\gamma}\left(l_{k}, y_{k}\right), C_{0}\right)<\frac{\varepsilon}{2} \\
& \left.\quad \text { and }\left\|T_{(A-\gamma B)_{0}}(\eta) y_{k}-y_{k}\right\| \leq \varepsilon\right\}
\end{aligned}
$$

where $\varepsilon^{*}:=\min (\varepsilon, \rho)$.

Set

$$
r_{k}:=\sup \left(I_{k}\right)>0 \text { and } l_{k+1}:=\min \left(l_{k}+\frac{r_{k}}{2}, \tau\right) .
$$

We define

$$
y_{k+1}=y_{k} \in C_{0} \text { if } l_{k+1}=\tau .
$$

Otherwise if $l_{k+1}=l_{k}+\frac{r_{k}}{2}<\tau$, then

$$
0<l_{k+1}-l_{k}=\frac{r_{k}}{2}<r_{k}
$$

hence

$$
l_{k+1}-l_{k} \in I_{k} .
$$

Thus, it follows that

$$
\frac{1}{l_{k+1}-l_{k}} d\left(T_{(A-\gamma B)_{0}}\left(l_{k+1}-l_{k}\right) y_{k}+S_{A-\gamma B}\left(l_{k+1}-l_{k}\right) F_{\gamma}\left(l_{k}, y_{k}\right), C_{0}\right)<\frac{\varepsilon}{2} .
$$

Therefore, we can find $y_{k+1} \in C_{0}$ satisfying

$$
\frac{1}{l_{k+1}-l_{k}}\left\|T_{(A-\gamma B)_{0}}\left(l_{k+1}-l_{k}\right) y_{k}+S_{A-\gamma B}\left(l_{k+1}-l_{k}\right) F_{\gamma}\left(l_{k}, y_{k}\right)-y_{k+1}\right\| \leq \frac{\varepsilon}{2} \text {. }
$$

Setting

$H_{k}:=\frac{1}{l_{k+1}-l_{k}}\left[y_{k+1}-T_{(A-\gamma B)_{0}}\left(l_{k+1}-l_{k}\right) y_{k}-S_{A-\gamma B}\left(l_{k+1}-l_{k}\right) F_{\gamma}\left(l_{k}, y_{k}\right)\right] \in X_{0}$.

Then it follows that

$$
H_{k} \in X_{0} \text { and }\left\|H_{k}\right\| \leq \frac{\varepsilon}{2}
$$

and

$y_{k+1}=T_{(A-\gamma B)_{0}}\left(l_{k+1}-l_{k}\right) y_{k}+S_{A-\gamma B}\left(l_{k+1}-l_{k}\right) F_{\gamma}\left(l_{k}, y_{k}\right)+\left(l_{k+1}-l_{k}\right) H_{k} \in C_{0}$. 
Lemma 4.7 Let Assumptions 2.1, 2.4, 3.1 and 4.4 be satisfied. Then the knots $\left(l_{k}, y_{k}\right), k \geq 0$ satisfy the following properties

(i) For all $k>m \geq 0$ we have

$$
\begin{aligned}
y_{k}= & T_{(A-\gamma B)_{0}}\left(l_{k}-l_{m}\right) y_{m}+\sum_{i=m}^{k-1}\left(l_{i+1}-l_{i}\right) T_{(A-\gamma B)_{0}}\left(l_{k}-l_{i+1}\right) H_{i} \\
& +\sum_{i=m}^{k-1} T_{(A-\gamma B)_{0}}\left(l_{k}-l_{i+1}\right) S_{A-\gamma B}\left(l_{i+1}-l_{i}\right) F_{\gamma}\left(l_{i}, y_{i}\right)
\end{aligned}
$$

(ii) $y_{k} \in B(0, \rho) \cap C_{0}$ for any $k \geq 0$.

(iii) For all $k>m \geq 0$ we have

$$
\left\|y_{k}-T_{(A-\gamma B)_{0}}\left(l_{k}-l_{m}\right) y_{m}\right\| \leq \Gamma \delta_{\gamma}\left(l_{k}-l_{m}\right)+\frac{\varepsilon}{2} M_{\gamma} e^{\omega_{\gamma}^{+}\left(l_{k}-l_{m}\right)}\left(l_{k}-l_{m}\right) .
$$

Proof. Proof of (i): Let $k>m \geq 0$ be given. Recall that for all $i=0, \ldots, k-1$ we have

$$
y_{i+1}=T_{(A-\gamma B)_{0}}\left(l_{i+1}-l_{i}\right) y_{i}+S_{A-\gamma B}\left(l_{i+1}-l_{i}\right) F_{\gamma}\left(l_{i}, y_{i}\right)+\left(l_{i+1}-l_{i}\right) H_{i} .
$$

Define the linear operator $L_{i}: X_{0} \rightarrow X_{0}$ by

$$
L_{i}:=T_{(A-\gamma B)_{0}}\left(l_{i+1}-l_{i}\right), i=0, \ldots, k-1
$$

Hence

$$
y_{i+1}=L_{i} y_{i}+S_{A-\gamma B}\left(l_{i+1}-l_{i}\right) F_{\gamma}\left(l_{i}, y_{i}\right)+\left(l_{i+1}-l_{i}\right) H_{i}, i=1, \ldots, k-1 .
$$

In order to use a variation of constants formula, we introduce the evolution family

$$
U(i, j)=L_{i-1} \cdots L_{j} \text { if } i>j \text { and } U(i, i)=I_{X_{0}} .
$$

Then it follows from the semigroup property that

$$
U(i, j)=T_{(A-\gamma B)_{0}}\left(l_{i}-l_{j}\right), \quad \text { if } i \geq j .
$$

By using a discrete variation of constants formula, we have for integers $k \geq m \geq$ 0

$$
\begin{aligned}
y_{k}= & U(k, m) y_{m}+\sum_{i=m}^{k-1} U(k, i+1)\left[S_{A-\gamma B}\left(l_{i+1}-l_{i}\right) F_{\gamma}\left(l_{i}, y_{i}\right)+\left(l_{i+1}-l_{i}\right) H_{i}\right] \\
= & T_{(A-\gamma B)_{0}}\left(l_{k}-l_{m}\right) y_{m}+\sum_{i=m}^{k-1}\left(l_{i+1}-l_{i}\right) T_{(A-\gamma B)_{0}}\left(l_{k}-l_{i+1}\right) H_{i} \\
& +\sum_{i=m}^{k-1} T_{(A-\gamma B)_{0}}\left(l_{k}-l_{i+1}\right) S_{A-\gamma B}\left(l_{i+1}-l_{i}\right) F_{\gamma}\left(l_{i}, y_{i}\right) .
\end{aligned}
$$


Proof of (ii): We will argue by recurrence. The property is true for $k=0$ since $y_{0}=x_{0} \in B(0, \rho) \cap C_{0}$. Assume that for $k \geq 1$

$$
y_{0}, \ldots, y_{k-1} \in B(0, \rho) \cap C_{0} .
$$

We are in a position to show that $y_{k} \in B(0, \rho) \cap C_{0}$. In view of (4.12), for any $m=0, \ldots, k-1$, we have

$y_{k}-T_{(A-\gamma B)_{0}}\left(l_{k}-l_{m}\right) y_{m}=\sum_{i=m}^{k-1} T_{(A-\gamma B)_{0}}\left(l_{k}-l_{i+1}\right)\left[S_{A-\gamma B}\left(l_{i+1}-l_{i}\right) F_{\gamma}\left(l_{i}, y_{i}\right)+\left(l_{i+1}-l_{i}\right) H_{i}\right]$.

Then it follows that

$$
\left\|y_{k}-T_{(A-\gamma B)_{0}}\left(l_{k}-l_{m}\right) y_{m}\right\| \leq\left\|W_{k, m}\right\|+\left\|Z_{k, m}\right\|,
$$

where

$$
W_{k, m}:=\sum_{i=m}^{k-1} T_{(A-\gamma B)_{0}}\left(l_{k}-l_{i+1}\right) S_{A-\gamma B}\left(l_{i+1}-l_{i}\right) F_{\gamma}\left(l_{i}, y_{i}\right)
$$

and

$$
Z_{k, m}:=\sum_{i=m}^{k-1}\left(l_{i+1}-l_{i}\right) T_{(A-\gamma B)_{0}}\left(l_{k}-l_{i+1}\right) H_{i} .
$$

Next, we do estimates of $W_{k, m}$ and $Z_{k, m}$. Since $H_{i} \in X_{0}$ and $\left\|H_{i}\right\| \leq \frac{\varepsilon}{2}$, for any $i=m, \ldots, k-1$, it is easy to obtain from (4.10) that

$$
\begin{aligned}
\left\|Z_{k, m}\right\| & \leq \sum_{i=m}^{k-1}\left(l_{i+1}-l_{i}\right) \frac{\varepsilon}{2} M_{\gamma} e^{\omega_{\gamma}\left(l_{i+1}-l_{i}\right)} \\
& \leq \frac{\varepsilon}{2} M_{\gamma} e^{\omega_{\gamma}^{+}\left(l_{k}-l_{m}\right)}\left(l_{k}-l_{m}\right),
\end{aligned}
$$

where

$$
\omega_{\gamma}^{+}=\max \left(0, \omega_{\gamma}\right) .
$$

In order to estimate $W_{k, m}$, we will rewrite it in a more convenient form. Using the following relationship

$$
T_{(A-\gamma B)_{0}}(\sigma) S_{A-\gamma B}(h)=S_{A-\gamma B}(\sigma+h)-S_{A-\gamma B}(\sigma), \forall \sigma \geq 0, \forall h \geq 0,
$$

we see that

$$
W_{k, m}=\sum_{i=m}^{k-1}\left[S_{A-\gamma B}\left(l_{k}-l_{i}\right)-S_{A-\gamma B}\left(l_{k}-l_{i+1}\right)\right] F_{\gamma}\left(l_{i}, y_{i}\right) .
$$

By Lemma 4.2 we have

$$
W_{k, m}=\left(S_{A-\gamma B} \diamond f_{\gamma}\left(l_{m}+\cdot\right)\right)\left(l_{k}-l_{m}\right)
$$


with step function

$f_{\gamma}(t)=F_{\gamma}\left(l_{i}, y_{i}\right), \forall t \in\left[l_{i}, l_{i+1}\right), i=m, \ldots, k-1$ and $f_{\gamma}\left(l_{k}\right)=F_{\gamma}\left(l_{k-1}, y_{k-1}\right)$.

Therefore by using the inequality (4.4) with $a=l_{m}$ and $b=l_{k}$ it follows that

$$
\begin{aligned}
\left\|W_{k, m}\right\| & =\left\|\left(S_{A-\gamma B} \diamond f_{\gamma}\left(l_{m}+\cdot\right)\right)\left(l_{k}-l_{m}\right)\right\| . \\
& \leq \delta_{\gamma}\left(l_{k}-l_{m}\right) \sup _{s \in\left[l_{m}, l_{k}\right]}\left\|f_{\gamma}(s)\right\| \\
& =\delta_{\gamma}\left(l_{k}-l_{m}\right) \max _{i=m, \ldots, k-1}\left\|F_{\gamma}\left(l_{i}, y_{i}\right)\right\| .
\end{aligned}
$$

By using (4.2) and the induction assumption, we deduce that

$$
\max _{i=m, \ldots, k-1}\left\|F_{\gamma}\left(l_{i}, y_{i}\right)\right\| \leq \Gamma .
$$

Then it follows from (4.13) and (4.14) that

$$
\left\|y_{k}-T_{(A-\gamma B)_{0}}\left(l_{k}-l_{m}\right) y_{m}\right\| \leq \Gamma \delta_{\gamma}\left(l_{k}-l_{m}\right)+\frac{\varepsilon}{2} M_{\gamma} e^{\omega_{\gamma}^{+}\left(l_{k}-l_{m}\right)}\left(l_{k}-l_{m}\right)
$$

for $m=0, \ldots, k-1$. To conclude the proof of (ii) we note that

$$
\left\|y_{k}-T_{(A-\gamma B)_{0}}\left(l_{k}-l_{0}\right) x_{0}\right\|=\left\|y_{k}-T_{(A-\gamma B)_{0}}\left(l_{k}\right) x_{0}\right\| \leq \Gamma \delta_{\gamma}\left(l_{k}\right)+M_{\gamma} e^{\omega_{\gamma}^{+} l_{k}} l_{k}
$$

and

$$
\begin{aligned}
\left\|y_{k}\right\| & \leq\left\|y_{k}-T_{(A-\gamma B)_{0}}\left(l_{k}\right) x_{0}\right\|+\left\|T_{(A-\gamma B)_{0}}\left(l_{k}\right) x_{0}\right\| \\
& \leq \Gamma \delta_{\gamma}\left(l_{k}\right)+M_{\gamma} e^{\omega_{\gamma}^{+} l_{k}} l_{k}+\left\|T_{(A-\gamma B)_{0}}\left(l_{k}\right) x_{0}\right\| .
\end{aligned}
$$

Since $l_{k} \in[0, \tau]$, the inequality (4.5) implies that $y_{k} \in B(0, \rho) \cap C_{0}$.

Proof of (iii): The proof follows the same lines in (ii).

Lemma 4.8 Let Assumptions 2.1, 2.4, 3.1 and 4.4 be satisfied. Then there exists an integer $n_{\varepsilon} \geq 1$ such that $l_{n_{\varepsilon}}=\tau$. That is to say that we have a finite number of knots $\left(l_{k}, y_{k}\right), k=0, \ldots, n_{\varepsilon}$ with

$$
0=l_{0}<l_{1}<\cdots<l_{n_{\varepsilon}-1}<l_{n_{\varepsilon}}=\tau \text { and } y_{0}, y_{1}, \ldots, y_{n_{\varepsilon}} \in C_{0}, y_{0}=x_{0} .
$$

Proof. We will use proof by contradiction. Assume that $l_{k}<\tau$ for all $k \geq 0$. That is to say that

$$
l_{k+1}=l_{k}+\frac{r_{k}}{2}, \forall k \geq 0 .
$$

Since the sequence is strictly increasing, there exists $l^{*} \leq \tau$ such that $l_{k} \rightarrow l^{*}$ as $k \rightarrow+\infty$ and $l_{k}<l^{*}$ for each $k \geq 0$. This also implies that

$$
\lim _{k \rightarrow+\infty} r_{k}=0
$$

In order to contradict (4.15), we will prove that there exists $k_{0}$ large enough and $\eta^{*}>0$ such that $\eta^{*} \in I_{k}$ for all $k \geq k_{0}$. This will mean that $r_{k}=\sup I_{k} \geq \eta^{*}>0$ for all $k \geq k_{0}$. 
Let us show that $\left\{y_{k}\right\}_{k>0}$ is a Cauchy sequence. To this end, we let $m \geq 0$ be arbitrary and $k \geq j>m$ be given. Then from Lemma 4.7, for all $k \geq j>m$, we have

$$
\begin{aligned}
\left\|y_{k}-y_{j}\right\| & \leq\left\|y_{k}-T_{(A-\gamma B)_{0}}\left(l_{k}-l_{m}\right) y_{m}\right\| \\
& +\left\|T_{(A-\gamma B)_{0}}\left(l_{k}-l_{m}\right) y_{m}-T_{(A-\gamma B)_{0}}\left(l_{j}-l_{m}\right) y_{m}\right\| \\
& +\left\|T_{(A-\gamma B)_{0}}\left(l_{j}-l_{m}\right) y_{m}-y_{j}\right\| \\
& \leq \Gamma \delta_{\gamma}\left(l_{k}-l_{m}\right)+M_{\gamma} e^{\omega_{\gamma}^{+}\left(l_{k}-l_{m}\right)}\left(l_{k}-l_{m}\right) \\
& +\left\|T_{(A-\gamma B)_{0}}\left(l_{k}-l_{m}\right) y_{m}-T_{(A-\gamma B)_{0}}\left(l_{j}-l_{m}\right) y_{m}\right\| \\
& +\Gamma \delta_{\gamma}\left(l_{j}-l_{m}\right)+M_{\gamma} e^{\omega_{\gamma}^{+}\left(l_{j}-l_{m}\right)}\left(l_{j}-l_{m}\right) .
\end{aligned}
$$

Then

$$
\limsup _{k, j \rightarrow+\infty}\left\|y_{k}-y_{j}\right\| \leq 2 \Gamma \delta_{\gamma}\left(l^{*}-l_{m}\right)+2 M_{\gamma} e^{\omega_{\gamma}^{+}\left(l^{*}-l_{m}\right)}\left(l^{*}-l_{m}\right) .
$$

Since $m$ is arbitrary and

$$
\lim _{m \rightarrow+\infty}\left[2 \Gamma \delta_{\gamma}\left(l^{*}-l_{m}\right)+2 M_{\gamma} e^{\omega_{\gamma}^{+}\left(l^{*}-l_{m}\right)}\left(l^{*}-l_{m}\right)\right]=0,
$$

we deduce that $\left(y_{k}\right)_{k \geq 0}$ is a Cauchy sequence in $B(0, \rho) \cap C_{0}$. Therefore there exists $y^{*} \in B(0, \rho) \cap \bar{C}_{0}$ such that

$$
\lim _{k \rightarrow+\infty} y_{k}=y^{*} \in C_{0} .
$$

Since $y^{*} \in C_{0}$ we have

$$
\lim _{h \rightarrow 0^{+}} \frac{1}{h} d\left(T_{(A-\gamma B)_{0}}(h) y^{*}+S_{A-\gamma B}(h) F_{\gamma}\left(l^{*}, y^{*}\right), C_{0}\right)=0 .
$$

By using the above limit, we can find $\eta^{*} \in\left(0, \frac{\varepsilon}{4}\right)$ small enough such that

$$
0<\eta^{*}<\frac{\varepsilon}{4}<\varepsilon^{*}
$$

and

$$
\frac{1}{\eta^{*}} d\left(T_{(A-\gamma B)_{0}}\left(\eta^{*}\right) y^{*}+S_{A-\gamma B}\left(\eta^{*}\right) F_{\gamma}\left(l^{*}, y^{*}\right), C_{0}\right) \leq \frac{\varepsilon}{4}
$$

and (by using the continuity of $\left.(l, y) \rightarrow T_{(A-\gamma B)_{0}}(l) y\right)$

$$
\left\|T_{(A-\gamma B)_{0}}\left(\eta^{*}\right) y^{*}-y^{*}\right\| \leq \frac{\varepsilon}{2}
$$

and (by using the continuity of $\left.(l, y) \rightarrow F_{\gamma}(l, y)\right)$

$$
\left|l^{*}-l\right| \leq 2 \eta^{*} \text { and }\left\|y-y^{*}\right\| \leq 2 \eta^{*} \Rightarrow\left\|F_{\gamma}(l, y)-F_{\gamma}\left(l^{*}, y^{*}\right)\right\| \leq \frac{\varepsilon}{2} .
$$


To obtain a contradiction, we will use the 1-Lipschitz continuity of $x \in X \rightarrow$ $d\left(x, C_{0}\right)$ combined with the continuity of $(l, y) \rightarrow F_{\gamma}(l, y)$ and $(l, y) \rightarrow T_{(A-\gamma B)_{0}}(l) y$ at $\left(l^{*}, y^{*}\right)$. Thus there exists $k_{0} \geq 0$ large enough such that for all $k \geq k_{0}$ one has

$$
\left\{\begin{array}{l}
\left\|F_{\gamma}\left(l_{k}, y_{k}\right)-F_{\gamma}\left(l^{*}, y^{*}\right)\right\| \leq \frac{\varepsilon}{2} \\
\left\|T_{(A-\gamma B)_{0}}\left(\eta^{*}\right) y_{k}-T_{(A-\gamma B)_{0}}\left(\eta^{*}\right) y^{*}\right\| \leq \frac{\varepsilon}{4} \\
\left\|y_{k}-y^{*}\right\| \leq \eta^{*} \text { and } 0<\left|l^{*}-l_{k}\right| \leq \eta^{*}
\end{array}\right.
$$

since $\eta^{*}$ is fixed and $y_{k} \rightarrow y^{*}$ and $l_{k} \rightarrow l^{*}$.

By using (4.17) and $y_{k} \rightarrow y^{*}$ and $l_{k} \rightarrow l^{*}$, we obtain for each $k \geq k_{0}$ (taking possibly $k_{0}$ larger)

$$
\frac{1}{\eta^{*}} d\left(T_{(A-\gamma B)_{0}}\left(\eta^{*}\right) y_{k}+S_{A-\gamma B}\left(\eta^{*}\right) F_{\gamma}\left(l_{k}, y_{k}\right), C_{0}\right)<\frac{\varepsilon}{2}, \forall k \geq k_{0} .
$$

Next we note that for any $k \geq k_{0}$

$$
0 \leq l-l_{k} \leq \eta^{*} \Rightarrow\left|l-l^{*}\right| \leq\left|l-l_{k}\right|+\left|l^{*}-l_{k}\right| \leq 2 \eta^{*}
$$

and

$$
\left\|y-y_{k}\right\| \leq \eta^{*} \Rightarrow\left\|y-y^{*}\right\| \leq\left\|y-y_{k}\right\|+\left\|y^{*}-y_{k}\right\| \leq 2 \eta^{*} .
$$

Combining (4.17)-(4.19) with (4.20), it follows that for any $k \geq k_{0}$

$$
\left\|F_{\gamma}(l, y)-F_{\gamma}\left(l_{k}, y_{k}\right)\right\| \leq\left\|F_{\gamma}(l, y)-F_{\gamma}\left(l^{*}, y^{*}\right)\right\|+\left\|F_{\gamma}\left(l^{*}, y^{*}\right)-F_{\gamma}\left(l_{k}, y_{k}\right)\right\| \leq \varepsilon
$$

whenever

$$
\left|l-l_{k}\right| \leq \eta^{*} \text { and }\left\|y-y_{k}\right\| \leq \eta^{*} .
$$

In view of (4.16), (4.17) and (4.20), we further have

$$
\begin{aligned}
\left\|T_{(A-\gamma B)_{0}}\left(\eta^{*}\right) y_{k}-y_{k}\right\| \leq & \left\|T_{(A-\gamma B)_{0}}\left(\eta^{*}\right) y_{k}-T_{(A-\gamma B)_{0}}\left(\eta^{*}\right) y^{*}\right\| \\
& +\left\|T_{(A-\gamma B)_{0}}\left(\eta^{*}\right) y^{*}-y^{*}\right\|+\left\|y^{*}-y_{k}\right\| \leq \varepsilon .
\end{aligned}
$$

Finally it follows from (4.21)-(4.24) that $0<\eta^{*} \in I_{k}$ for all $k \geq k_{0}$ which contradicts (4.15).

Construction of the approximate solution: Recall that from property (i) of Lemma 4.7 we have for each $m=0, \ldots, k-1$ and each $k \geq 1$

$$
\begin{aligned}
y_{k}= & T_{(A-\gamma B)_{0}}\left(l_{k}-l_{m}\right) y_{m}+\sum_{i=m}^{k-1}\left(l_{i+1}-l_{i}\right) T_{(A-\gamma B)_{0}}\left(l_{k}-l_{i+1}\right) H_{i} \\
& +\sum_{i=m}^{k-1} T_{(A-\gamma B)_{0}}\left(l_{k}-l_{i+1}\right) S_{A-\gamma B}\left(l_{i+1}-l_{i}\right) F_{\gamma}\left(l_{i}, y_{i}\right) .
\end{aligned}
$$


For each $t \in\left[l_{k}, l_{k+1}\right]$ and each $k=0, \ldots, n_{\varepsilon}-1$, we set

$$
\begin{aligned}
u_{\varepsilon}(t):= & T_{(A-\gamma B)_{0}}\left(t-l_{0}\right) y_{0}+S_{A-\gamma B}\left(t-l_{k}\right) F_{\gamma}\left(l_{k}, y_{k}\right)+\left(t-l_{k}\right) H_{k} \\
& +\sum_{i=0}^{k-1}\left(l_{i+1}-l_{i}\right) T_{(A-\gamma B)_{0}}\left(t-l_{i+1}\right) H_{i} \\
& +\sum_{i=0}^{k-1} T_{(A-\gamma B)_{0}}\left(t-l_{i+1}\right) S_{A-\gamma B}\left(l_{i+1}-l_{i}\right) F_{\gamma}\left(l_{i}, y_{i}\right)
\end{aligned}
$$

with the convention

$$
\sum_{i=m}^{p}=0 \text { if } p<m
$$

By using the semigroup property for $t \rightarrow T_{(A-\gamma B)_{0}}(t)$, we deduce from (4.25) and (4.26) that

$u_{\varepsilon}(t)=T_{(A-\gamma B)_{0}}\left(t-l_{k}\right) y_{k}+S_{A-\gamma B}\left(t-l_{k}\right) F_{\gamma}\left(l_{k}, y_{k}\right)+\left(t-l_{k}\right) H_{k}, \forall t \in\left[l_{k}, l_{k+1}\right]$.

Then it is clear that $u_{\varepsilon}(t)$ is well defined and continuous from $[0, \tau]$ into $X_{0}$ and

$$
u_{\varepsilon}\left(l_{k}\right)=y_{k}, \forall k=0, \ldots, n_{\varepsilon} .
$$

Next we rewrite $u_{\varepsilon}(t)$ into a form that will be convenient for our subsequent discussions. By using the relationship

$$
S_{A-\gamma B}(h+\sigma)-S_{A-\gamma B}(\sigma)=T_{(A-\gamma B)_{0}}(\sigma) S_{A-\gamma B}(h), \forall h \geq 0, \forall \sigma \geq 0
$$

one can rewrite from (4.26) the formula of $u_{\varepsilon}$ as

$$
\begin{aligned}
u_{\varepsilon}(t)= & T_{(A-\gamma B)_{0}}\left(t-l_{0}\right) y_{0}+S_{A-\gamma B}\left(t-l_{k}\right) F_{\gamma}\left(l_{k}, y_{k}\right)+\left(t-l_{k}\right) H_{k} \\
& +\sum_{i=0}^{k-1}\left(l_{i+1}-l_{i}\right) T_{(A-\gamma B)_{0}}\left(t-l_{i+1}\right) H_{i} \\
& +\sum_{i=0}^{k-1}\left[S_{A-\gamma B}\left(t-l_{i+1}\right)-S_{A-\gamma B}\left(t-l_{i}\right)\right] F_{\gamma}\left(l_{i}, y_{i}\right), \forall t \in\left[l_{k}, l_{k+1}\right] .
\end{aligned}
$$

Setting

$$
f_{\gamma}(t)=F_{\gamma}\left(l_{i}, y_{i}\right), \forall t \in\left[l_{i}, l_{i+1}\right), i=0, \ldots, n_{\varepsilon}-1, f_{\gamma}\left(l_{n_{\varepsilon}}\right)=F_{\gamma}\left(l_{n_{\varepsilon}-1}, y_{n_{\varepsilon}-1}\right)
$$

and remembering that $y_{0}=x_{0}$, by Lemma 4.2 we obtain for each $t \in\left[l_{k}, l_{k+1}\right]$,

$$
\begin{aligned}
u_{\varepsilon}(t)= & T_{(A-\gamma B)_{0}}\left(t-l_{0}\right) x_{0}+\left(S_{A-\gamma B} \diamond f_{\gamma}\left(l_{0}+\cdot\right)\right)\left(t-l_{0}\right) \\
& +\left(t-l_{k}\right) H_{k}+\sum_{i=0}^{k-1}\left(l_{i+1}-l_{i}\right) T_{(A-\gamma B)_{0}}\left(t-l_{i+1}\right) H_{i} .
\end{aligned}
$$

Similar arguments also gives for any $t \in\left[l_{k}, l_{k+1}\right]$ and each integer $m \in[0, k]$

$$
\begin{aligned}
u_{\varepsilon}(t)= & T_{(A-\gamma B)_{0}}\left(t-l_{m}\right) y_{m}+\left(S_{A-\gamma B} \diamond f_{\gamma}\left(l_{m}+\cdot\right)\right)\left(t-l_{m}\right) \\
& +\left(t-l_{k}\right) H_{k}+\sum_{i=m}^{k-1}\left(l_{i+1}-l_{i}\right) T_{(A-\gamma B)_{0}}\left(t-l_{i+1}\right) H_{i} .
\end{aligned}
$$


By using again (4.2), we also have the following estimate that for any $t \in\left[l_{m}, l_{k}\right]$ with $k \geq m$,

$$
\left\|\left(S_{A-\gamma B} \diamond f_{\gamma}\left(l_{m}+\cdot\right)\right)\left(t-l_{m}\right)\right\| \leq \Gamma \delta_{\gamma}\left(t-l_{m}\right) .
$$

Lemma 4.9 Let Assumptions 2.1, 2.4, 3.1 and 4.4 be satisfied. Then the approximate solution $u_{\varepsilon}(t)$ in (4.29) satisfies the following properties

(i) There exits a constant $\hat{M}_{0}>0$ such that

$$
\left\|u_{\varepsilon}(t)-y_{k}\right\| \leq \hat{M}_{0}\left(\varepsilon+\delta_{\gamma}(\varepsilon)\right), \forall t \in\left[l_{k}, l_{k+1}\right]
$$

with $k=0, \ldots, n_{\varepsilon}-1$.

(ii) $u_{\varepsilon}(t) \in B(0, \rho), \forall t \in[0, \tau]$.

(iii) There exists a constant $\hat{M}_{1}>0$ such that for all $t \in[0, \tau]$

$$
\| u_{\varepsilon}(t)-T_{(A-\gamma B)_{0}}(t) x_{0}-\left(S_{A-\gamma B} \diamond F_{\gamma}\left(\cdot, u_{\varepsilon}(\cdot)\right)(t) \| \leq \hat{M}_{1}\left(\varepsilon+\delta_{\gamma}(\varepsilon)\right) .\right.
$$

Proof. We first prove that, for each $t \in\left[l_{m}, l_{p}\right]$ with $p \geq m \geq 0$ and each $\bar{y} \in X_{0}$, we have

$\left\|u_{\varepsilon}(t)-\bar{y}\right\| \leq\left\|T_{(A-\gamma B)_{0}}\left(t-l_{m}\right) y_{m}-\bar{y}\right\|+\Gamma \delta_{\gamma}\left(t-l_{m}\right)+\frac{\varepsilon}{2} M_{\gamma}\left(t-l_{m}\right) e^{\omega_{\gamma}^{+}\left(t-l_{m}\right)}$.

Let $p>m \geq 0$ be given. From (4.30) we have

$$
\begin{aligned}
u_{\varepsilon}(t)-\bar{y}= & T_{(A-\gamma B)_{0}}\left(t-l_{m}\right) y_{m}-\bar{y}+\left(S_{A-\gamma B} \diamond f_{\gamma}\left(l_{m}+\cdot\right)\right)\left(t-l_{m}\right) \\
& +\left(t-l_{k}\right) H_{k}+\sum_{i=m}^{k-1}\left(l_{i+1}-l_{i}\right) T_{(A-\gamma B)_{0}}\left(t-l_{i+1}\right) H_{i}, \forall t \in\left[l_{k}, l_{k+1}\right]
\end{aligned}
$$

with $m \leq k \leq p-1$. Hence

$$
\begin{aligned}
\left\|u_{\varepsilon}(t)-\bar{y}\right\| \leq & \left\|T_{(A-\gamma B)_{0}}\left(t-l_{m}\right) y_{m}-\bar{y}\right\|+\left\|\left(S_{A-\gamma B} \diamond f_{\gamma}\left(l_{m}+\cdot\right)\right)\left(t-l_{m}\right)\right\| \\
& +\left(t-l_{k}\right)\left\|H_{k}\right\|+\sum_{i=m}^{k-1}\left(l_{i+1}-l_{i}\right)\left\|T_{(A-\gamma B)_{0}}\left(t-l_{i+1}\right) H_{i}\right\|, \quad \forall t \in\left[l_{k}, l_{k+1}\right]
\end{aligned}
$$

with $m \leq k \leq p-1$. In view of (4.31), and

$$
H_{i} \in X_{0} \text { and }\left\|H_{i}\right\| \leq \frac{\varepsilon}{2}, i=0, \ldots, n_{\varepsilon},
$$

we see that, for any $t \in\left[l_{k}, l_{k+1}\right]$ with $m \leq k \leq p-1$,

$$
\begin{aligned}
\left\|u_{\varepsilon}(t)-\bar{y}\right\| \leq & \left\|T_{(A-\gamma B)_{0}}\left(t-l_{m}\right) y_{m}-\bar{y}\right\|+\Gamma \delta_{\gamma}\left(t-l_{m}\right)+\left(t-l_{k}\right) \frac{\varepsilon}{2} \\
& +\sum_{i=m}^{k-1} M_{\gamma} e^{\omega_{\gamma}\left(t-l_{i+1}\right)} \frac{\varepsilon}{2}\left(l_{i+1}-l_{i}\right) \\
\leq & \left\|T_{(A-\gamma B)_{0}}\left(t-l_{m}\right) y_{m}-\bar{y}\right\|+\Gamma \delta_{\gamma}\left(t-l_{m}\right)+\frac{\varepsilon}{2} M_{\gamma}\left(t-l_{m}\right) e^{\omega_{\gamma}^{+}\left(t-l_{m}\right)} \\
\leq & \left\|T_{(A-\gamma B)_{0}}\left(t-l_{m}\right) y_{m}-\bar{y}\right\|+\Gamma \delta_{\gamma}\left(t-l_{m}\right)+\frac{\varepsilon}{2} M_{\gamma}\left(t-l_{m}\right) e^{\omega_{\gamma}^{+}\left(t-l_{m}\right)},
\end{aligned}
$$


which proves (4.33).

Proof of (i): By using (4.33) with $m=k, p=k+1$ and $\bar{y}=y_{k}$, for each $t \in\left[l_{k}, l_{k+1}\right]$, it follows that

$\left\|u_{\varepsilon}(t)-y_{k}\right\| \leq\left\|T_{(A-\gamma B)_{0}}\left(t-l_{k}\right) y_{k}-y_{k}\right\|+\Gamma \delta_{\gamma}\left(t-l_{k}\right)+\frac{\varepsilon}{2} M_{\gamma}\left(t-l_{k}\right) e^{\omega_{\gamma}^{+}\left(t-l_{k}\right)}$.

Observing that

$$
t \in\left[l_{k}, l_{k+1}\right] \Rightarrow t-l_{k} \leq l_{k+1}-l_{k} \leq \frac{r_{k}}{2}<r_{k} \leq \varepsilon \Rightarrow t-l_{k} \in I_{k}
$$

where $I_{k}$ and $r_{k}$ are defined respectively in (4.7) and (4.8). Then we deduce that

$$
\left\|T_{(A-\gamma B)_{0}}\left(t-l_{k}\right) y_{k}-y_{k}\right\| \leq \varepsilon, \forall t \in\left[l_{k}, l_{k+1}\right]
$$

and

$$
\left\|u_{\varepsilon}(t)-y_{k}\right\| \leq \varepsilon+\Gamma \delta_{\gamma}(\varepsilon)+\frac{\varepsilon}{2} M_{\gamma} \varepsilon e^{\omega_{\gamma}^{+} \varepsilon}, \forall t \in\left[l_{k}, l_{k+1}\right] .
$$

This proves (i).

Proof of (ii): In view of (4.33) with $m=0, p=n_{\varepsilon}$ and $\bar{y}=0$, and using the fact $l_{0}=0$ and $y_{0}=x_{0}$, we deduce that

$$
\left\|u_{\varepsilon}(t)\right\| \leq\left\|T_{(A-\gamma B)_{0}}(t) x_{0}\right\|+\Gamma \delta_{\gamma}(t)+M_{\gamma} e^{\omega_{\gamma}^{+} t} t, \forall t \in[0, \tau] .
$$

Then the fact $0 \leq t \leq \tau$ together with the inequality (4.5) imply that

$$
\left\|u_{\varepsilon}(t)\right\| \leq \rho, \forall t \in[0, \tau] .
$$

Proof of (iii): Let

$$
v_{\varepsilon}(t)=u_{\varepsilon}(t)-T_{(A-\gamma B)_{0}}(t) x_{0}-\left(S_{A-\gamma B} \diamond F_{\gamma}\left(\cdot, u_{\varepsilon}(\cdot)\right)(t), \forall t \in[0, \tau] .\right.
$$

We further define

$$
g_{\gamma}(t):=f_{\gamma}(t)-F_{\gamma}\left(t, u_{\varepsilon}(t)\right), \forall t \in[0, \tau]
$$

or equivalently

$g_{\gamma}(t)= \begin{cases}F_{\gamma}\left(l_{k}, y_{k}\right)-F_{\gamma}\left(t, u_{\varepsilon}(t)\right) & \text { if } \quad t \in\left[l_{k}, l_{k+1}\right), k=0, \ldots, n_{\varepsilon}-1 \\ F_{\gamma}\left(l_{n_{\varepsilon}-1}, y_{n_{\varepsilon}-1}\right)-F_{\gamma}\left(l_{n_{\varepsilon}}, y_{n_{\varepsilon}}\right) & \text { if } \quad t=l_{n_{\varepsilon}} .\end{cases}$

where $f_{\gamma}$ is defined in (4.28) and $n_{\varepsilon}$ has been defined in Lemma 4.8.

Then using (4.30) we get

$v_{\varepsilon}(t)=\left(S_{A-\gamma B} \diamond g_{\gamma}(\cdot)\right)(t)+\left(t-l_{k}\right) H_{k}+\sum_{i=0}^{k-1}\left(l_{i+1}-l_{i}\right) T_{(A-\gamma B)_{0}}\left(t-l_{i+1}\right) H_{i}, \forall t \in\left[l_{k}, l_{k+1}\right]$.

Since $g_{\gamma} \in \operatorname{Reg}([0, \tau], X)$, it follows that

$$
\begin{aligned}
\left\|v_{\varepsilon}(t)\right\| & \leq \delta_{\gamma}(t) \sup _{s \in[0, t]}\left\|g_{\gamma}(s)\right\|+\frac{\varepsilon}{2} M_{\gamma}\left(t-l_{0}\right) e^{\omega_{\gamma}^{+} t} \\
& \leq \delta_{\gamma}(\tau) \sup _{s \in[0, t]}\left\|g_{\gamma}(s)\right\|+\frac{\varepsilon}{2} M_{\gamma} \tau e^{\omega_{\gamma}^{+} \tau} .
\end{aligned}
$$


Therefore one can obtain (4.32) by estimating

$$
\sup _{s \in[0, t]}\left\|g_{\gamma}(s)\right\|, \forall t \in[0, \tau] .
$$

In view of (4.35), it follows that

$$
\left\|g_{\gamma}(t)\right\| \leq\left\|F_{\gamma}\left(l_{k}, y_{k}\right)-F_{\gamma}\left(t, y_{k}\right)\right\|+\left\|F_{\gamma}\left(t, y_{k}\right)-F_{\gamma}\left(t, u_{\varepsilon}(t)\right)\right\|, t \in\left[l_{k}, l_{k+1}\right]
$$

with $k=0, \ldots, n_{\varepsilon}$. Observing that if $t \in\left[l_{k}, l_{k+1}\right]$, then

$$
t-l_{k} \leq l_{k+1}-l_{k} \leq \frac{r_{k}}{2}<r_{k} \leq \rho \Rightarrow t-l_{k} \in I_{k} \text { and } t \in[0, \rho]
$$

where $I_{k}$ and $r_{k}$ are defined respectively in (4.7) and (4.8). This observation together with the fact

$$
u_{\varepsilon}(t) \in B(0, \rho), \forall t \in[0, \tau]
$$

implying that

$$
\left\|g_{\gamma}(t)\right\| \leq \varepsilon+\Lambda\left\|y_{k}-u_{\varepsilon}(t)\right\|, \forall t \in\left[l_{k}, l_{k+1}\right] .
$$

Finally we infer from (4.34) that

$$
\left\|g_{\gamma}(t)\right\| \leq \varepsilon+\Lambda\left[\varepsilon+\Gamma \delta_{\gamma}(\varepsilon)+\frac{\varepsilon}{2} M_{\gamma} \varepsilon e^{\omega_{\gamma}^{+} \varepsilon}\right], \forall t \in\left[l_{k}, l_{k+1}\right] .
$$

The result follows.

Existence of solution in $C_{0}$ : At this stage, the approximated solution $t \rightarrow$ $u_{\varepsilon}(t)$ only belongs to $C_{0}$ for $t=l_{k}$ (since $u\left(l_{k}\right)=y_{k} \in C_{0}$ ). In this last part of the proof, we take the limit when $\varepsilon \rightarrow 0$ and after proving that the limit exits (by using Cauchy sequences), we will prove that the limit solution takes his value in $C_{0}$.

We first prove that the approximated solution $\left(u_{\varepsilon}\right)_{\varepsilon \in\left(0, \varepsilon^{*}\right)}$ forms a Cauchy sequence in $C\left([0, \tau], X_{0}\right)$ and its limit is a solution of system (1.1). Indeed, by using property (iii) of Lemma 4.9, we have

$$
\left\|u_{\varepsilon}(t)-u_{\sigma}(t)\right\| \leq \hat{M}_{1}\left[\varepsilon+\delta_{\gamma}(\varepsilon)+\sigma+\delta_{\gamma}(\sigma)\right]+\delta_{\gamma}(t) \sup _{s \in[0, t]}\left\|F_{\gamma}\left(s, u_{\varepsilon}(s)\right)-F_{\gamma}\left(s, u_{\sigma}(s)\right)\right\| .
$$

Since

$$
u_{\varepsilon}(t), u_{\sigma}(t) \in B(0, \rho), \forall t \in[0, \tau], 0<\tau \leq \rho,
$$

we obtain

$$
\left\|u_{\varepsilon}(t)-u_{\sigma}(t)\right\| \leq \hat{M}_{1}\left[\varepsilon+\delta_{\gamma}(\varepsilon)+\sigma+\delta_{\gamma}(\sigma)\right]+\delta_{\gamma}(\tau) \Lambda \sup _{s \in[0, \tau]}\left\|u_{\varepsilon}(s)-u_{\sigma}(s)\right\|, \forall t \in[0, \tau] .
$$

In view of (4.6), we have $0<\delta_{\gamma}(\tau) \Lambda<1$, and hence,

$$
\sup _{t \in[0, \tau]}\left\|u_{\varepsilon}(t)-u_{\sigma}(t)\right\| \leq \frac{\hat{M}_{1}}{1-\delta_{\gamma}(\tau) \Lambda}\left[\varepsilon+\delta_{\gamma}(\varepsilon)+\sigma+\delta_{\gamma}(\sigma)\right] .
$$


Therefore $\left(u_{\varepsilon}\right)_{\varepsilon \in\left(0, \varepsilon^{*}\right)} \in C\left([0, \tau], X_{0}\right)$ is a Cauchy sequence in $C\left([0, \tau], X_{0}\right)$ endowed with the supremum norm. Then there exists $u \in C\left([0, \tau], X_{0}\right)$ such that

$$
\lim _{\varepsilon \rightarrow 0^{+}} \sup _{t \in[0, \tau]}\left\|u_{\varepsilon}(t)-u(t)\right\|=0
$$

Letting $\varepsilon$ tend to zero in (4.32), it is straightforward that

$$
\begin{aligned}
u(t) & =T_{(A-\gamma B)_{0}}(t) x_{0}+\left(S_{A-\gamma B} \diamond F_{\gamma}(\cdot, u(\cdot))(t)\right. \\
& =T_{A_{0}}(t) x_{0}+\left(S_{A} \diamond F(\cdot, u(\cdot))(t), \forall t \in[0, \tau], \forall t \in[0, \tau] .\right.
\end{aligned}
$$

That is to say that $u \in C\left([0, \tau], X_{0}\right)$ is a mild solution of $(1.1)$ in $[0, \tau]$. Finally using property (i) of Lemma 4.9, we see that

$$
d\left(u_{\varepsilon}(t), C_{0}\right) \leq \hat{M}_{0}\left(\varepsilon+\delta_{\gamma}(\varepsilon)\right), \forall t \in[0, \tau] \Rightarrow \lim _{\varepsilon \rightarrow 0^{+}} d\left(u_{\varepsilon}(t), C_{0}\right)=0, \forall t \in[0, \tau] .
$$

By the continuity of $x \in X_{0} \mapsto d\left(x, C_{0}\right)$, we further see that

$$
d\left(u(t), C_{0}\right)=\lim _{\varepsilon \rightarrow 0^{+}} d\left(u_{\varepsilon}(t), C_{0}\right), \forall t \in[0, \tau] \Rightarrow u(t) \in C_{0}, \forall t \in[0, \tau] .
$$

\section{Applications to age structured models}

We will consider a generalization of the one dimensional model presented in [17]. The model considered is the following

$$
\left\{\begin{array}{l}
\frac{\partial u(t, a)}{\partial t}+\frac{\partial u(t, a)}{\partial a}=-\mu(a) u(t, a)(\kappa-\Theta(u(t, a))) \\
u(t, 0)=\int_{0}^{+\infty} \beta(a) u(t, a)(\kappa-\Theta(u(t, a))) d a \\
u(0, .)=u_{0} \in \mathrm{L}_{+}^{p}\left(\mathbb{R}_{+}, \mathbb{R}^{n}\right), p \in[1,+\infty)
\end{array}\right.
$$

where we have set

$$
\Theta(x)=\sum_{k=0}^{n} x_{k}, \forall x \in \mathbb{R}^{n}
$$

and assume that $\kappa>0, \beta, \mu \in \mathrm{L}_{+}^{\infty}\left(\mathbb{R}_{+}, \mathbb{R}\right)$ with $\frac{1}{p}+\frac{1}{q}=1$ and

$$
\beta(a)=0, \forall a \geq a_{\dagger} \text { and } \mu(a) \geq \mu_{-}>0, \forall a \geq 0 .
$$

It is important to note that the model (5.1) is not well defined in $\mathrm{L}^{p}\left(\mathbb{R}_{+}, \mathbb{R}^{n}\right)$ however it does in a proper subset of $\mathrm{L}^{p}\left(\mathbb{R}_{+}, \mathbb{R}^{n}\right)$ namely

$$
C=\left\{\varphi \in \mathrm{L}_{+}^{p}\left(\mathbb{R}_{+}, \mathbb{R}^{n}\right): 0 \leq \Theta(\varphi(a)) \leq \kappa \text { for a.e. } a \geq 0\right\} .
$$

Truncated system: The interest of our result is that we will be able to demonstrate the existence of solutions for initial data in $C$. To do so we introduce the following truncation function $\chi: \mathbb{R} \rightarrow[0, \kappa]$ defined by

$$
\chi(s)=\min \left(k, s^{+}\right), \forall s \in \mathbb{R}
$$


and we set for each $i=1, \ldots, n$

$$
\left\{\begin{array}{l}
\frac{\partial u_{i}(t, a)}{\partial t}+\frac{\partial u_{i}(t, a)}{\partial a}=-\mu(a) \chi\left(u_{i}(t, a)\right) \chi(\kappa-\Theta(u(t, a))) \\
u_{i}(t, 0)=\int_{0}^{+\infty} \beta(a) \chi\left(u_{i}(t, a)\right) \chi(\kappa-\Theta(u(t, a))) d a \\
u_{i}(0, .)=u_{i 0} \in \mathrm{L}_{+}^{p}\left(\mathbb{R}_{+}, \mathbb{R}\right), p \in[1,+\infty)
\end{array}\right.
$$

which is well defined in $\mathrm{L}_{+}^{p}\left(\mathbb{R}_{+}, \mathbb{R}^{n}\right)$. The idea is to prove that for each $\varphi \in C$ there exists a unique mild solution of (5.3) lying in $C$ and since the two systems coincide in $C$ the result follows.

Abstract reformulation: Set

$$
X=\mathbb{R}^{n} \times \mathrm{L}^{p}\left(\mathbb{R}_{+}, \mathbb{R}^{n}\right)
$$

endowed with the usual product norm. Consider the linear operator $A: D(A) \subset$ $X \rightarrow X$

$$
A\left(\begin{array}{c}
0 \mathbb{R}^{n} \\
\varphi
\end{array}\right)=\left(\begin{array}{c}
-\varphi(0) \\
-\varphi^{\prime}
\end{array}\right)
$$

and

$$
D(A)=\left\{0_{\mathbb{R}^{n}}\right\} \times \mathrm{W}^{1, p}\left(\mathbb{R}_{+}, \mathbb{R}^{n}\right)
$$

and note that the closure of the domain of $A$ is

$$
X_{0}:=\overline{D(A)}=\left\{0_{\mathbb{R}^{n}}\right\} \times \mathrm{L}^{p}\left(\mathbb{R}_{+}, \mathbb{R}^{n}\right)
$$

Consider the non linear maps $F_{0}: \mathrm{L}^{p}\left(\mathbb{R}_{+}, \mathbb{R}^{n}\right) \rightarrow \mathbb{R}^{n}$ and $F_{1}: \mathrm{L}^{p}\left(\mathbb{R}_{+}, \mathbb{R}^{n}\right) \rightarrow$ $\mathrm{L}^{p}\left(\mathbb{R}_{+}, \mathbb{R}^{n}\right)$ defined respectively for each $i=1, \ldots, n$ by

$$
F_{0}(\varphi)_{i}=\int_{0}^{+\infty} \beta(a) \chi\left(\varphi_{i}(a)\right) \chi(\kappa-\Theta(\varphi(a))) d a, \text { for a.e } a \geq 0
$$

and

$$
F_{1}(\varphi)_{i}(a)=-\mu(a) \chi\left(\varphi_{i}(a)\right) \chi(\kappa-\Theta(\varphi(a))), \text { for a.e } a \geq 0 .
$$

Next we consider $F: X_{0} \rightarrow X$ defined by

$$
F\left(\begin{array}{c}
0_{\mathbb{R}^{n}} \\
\varphi
\end{array}\right)=\left(\begin{array}{c}
F_{0}(\varphi) \\
F_{1}(\varphi)
\end{array}\right)
$$

By identifying $u(t,$.$) with v(t):=\left(\begin{array}{c}0 \mathbb{R}^{n} \\ u(t, .)\end{array}\right)$ we can rewrite the partial differential equation (5.1) as the following abstract Cauchy problem

$$
v^{\prime}(t)=A v(t)+F(v(t)), \text { for } t \geq 0, v(0)=\left(\begin{array}{c}
0_{\mathbb{R}^{n}} \\
u_{0}
\end{array}\right) \in X_{0} .
$$

It is well known that the linear operator $A: D(A) \subset X_{0} \rightarrow X_{0}$ is not HilleYosida for $p>1$ but fulfill the conditions of Assumption 2.1 (see [8, Section 
6]). By using similar arguments in [8] one can also show that Assumption 2.4 is satisfied. It can be easily checked that $F$ is Lipschitz on bounded sets of $X_{0}$. Therefore in what follow we will only verify that Assumption 4.4 is satisfied. We consider the following closed subset as a candidate for the application of our results

$$
\mathcal{C}_{0}=\left\{0_{\mathbb{R}^{n}}\right\} \times C .
$$

In order to verify Assumption 4.4 we will first determine the strongly continuous semigroup $\left\{T_{A_{0}}(t)\right\}_{t \geq 0} \subset \mathcal{L}\left(X_{0}\right)$ generated by $A_{0}$ the part of $A$ in $X_{0}$ and the integrated semigroup $\left\{S_{A}(t)\right\}_{t \geq 0} \subset \mathcal{L}(X)$ generated by $A$. Indeed $\left\{T_{A_{0}}(t)\right\}_{t \geq 0} \subset \mathcal{L}\left(X_{0}\right)$ is given by

$$
T_{A_{0}}(t)\left(\begin{array}{c}
0_{\mathbb{R}^{n}} \\
\varphi
\end{array}\right)=\left(\begin{array}{c}
0_{\mathbb{R}^{n}} \\
\widehat{T}_{A_{0}}(t)(\varphi)
\end{array}\right), \forall\left(\begin{array}{c}
0_{\mathbb{R}^{n}} \\
\varphi
\end{array}\right) \in X_{0}
$$

with $t \rightarrow \widehat{T}_{A_{0}}(t)(\varphi)$ the unique continuous mild solution of the partial differential equation

$$
\left\{\begin{array}{l}
\frac{\partial u(t, a)}{\partial t}+\frac{\partial u(t, a)}{\partial a}=0, t>0, a>0 \\
u(t, 0)=0, t>0 \\
u(0, .)=\varphi \in \mathrm{L}^{p}\left(\mathbb{R}_{+}, \mathbb{R}^{n}\right) .
\end{array}\right.
$$

Thus integrating along the characteristics yields

$$
\widehat{T}_{A_{0}}(t)(\varphi)(a)=\left\{\begin{array}{l}
\varphi(a-t), \text { if } a>t, \\
0, \text { if } a<t,
\end{array}\right.
$$

which can be rewritten into the more condensed form

$$
\widehat{T}_{A_{0}}(t)(\varphi)(a)=H(a-t) \varphi(a-t), \forall t \geq 0, \text { for a.e. } a \geq 0
$$

where the map $\varphi(a)$ is understood as its extension by 0 for almost every $a<0$ and $a \rightarrow H(a)$ is the Heaviside function defined by

$$
H(a)=1 \text { if } a \geq 0 \text { and } H(a)=0 \text {, if } a<0 .
$$

Furthermore the integrated semigroup generated by $A$ is given by

$$
S_{A}(t)\left(\begin{array}{c}
x \\
\varphi
\end{array}\right)=\left(\begin{array}{c}
0 \mathbb{R}^{n} \\
\widehat{S}_{A}(t)(x, \varphi)
\end{array}\right), \forall\left(\begin{array}{c}
x \\
\varphi
\end{array}\right) \in X
$$

with $t \rightarrow \widehat{S}_{A}(t)(x, \varphi)$ the unique mild solution of the partial differential equation

$$
\left\{\begin{array}{l}
\frac{\partial u(t, a)}{\partial t}+\frac{\partial u(t, a)}{\partial a}=\varphi(a), t>0, a>0, \\
u(t, 0)=x, t>0 \\
u(0, .)=0_{\mathrm{L}^{p}}
\end{array}\right.
$$

which is obtained by integrating along the characteristics as follow

$$
\widehat{S}_{A}(t)(x, 0)(a)=\left\{\begin{array}{l}
x, \text { if } t>a, \\
0, \text { if } t<a,
\end{array}\right.
$$


and

$$
\widehat{S}_{A}(t)(0, \varphi)(a)=\left(\int_{0}^{t} \widehat{T}_{A_{0}}(l)(\varphi) d l\right)(a)
$$

therefore (since by linearity $\widehat{S}_{A}(t)(x, \varphi)=\widehat{S}_{A}(t)(x, 0)+\widehat{S}_{A}(t)(0, \varphi)$ we obtain $\widehat{S}_{A}(t)(x, \varphi)(a)=(1-H(a-t)) x+\left(\int_{0}^{t} \widehat{T}_{A_{0}}(l)(\varphi) d l\right)(a), \forall t \geq 0$, for a.e. $a \geq 0$.

The following lemma will allows us to give a more explicit form of (5.8).

Lemma 5.1 For each $t \geq 0$ we have

$$
\widehat{S}_{A}(t)(0, \varphi)(a)=\int_{0}^{t} \widehat{T}_{A_{0}}(l)(\varphi)(a) d l=\int_{0}^{t} H(a-l) \varphi(a-l) d l, \text { for a.e. } a \geq 0
$$

where $H$ is the Heaviside function. Moreover we have

$\widehat{S}_{A}(t)(x, \varphi)(a)=(1-H(a-t)) x+\int_{0}^{t} H(a-l) \varphi(a-l) d l, \forall t \geq 0, \quad$ a.e. $a \geq 0$.

Proof. Let $x^{*}: L^{p}\left(\mathbb{R}_{+}, \mathbb{R}^{n}\right) \rightarrow \mathbb{R}$ any linear continuous functional. Then by the Riesz representation theorem there exists a unique $\psi \in L^{q}\left(\mathbb{R}_{+}, \mathbb{R}^{n}\right)$ with $\frac{1}{p}+\frac{1}{q}=1$ such that

$$
x^{*}(\phi)=\int_{0}^{+\infty} \psi(a) \phi(a) d a, \forall \phi \in L^{p}\left(\mathbb{R}_{+}, \mathbb{R}^{n}\right) .
$$

Therefore we have by using Fubini's theorem for each $t \geq 0$

$$
\begin{aligned}
x^{*}\left(\widehat{S}_{A}(t)(0, \varphi)\right) & =x^{*}\left(\int_{0}^{t} \widehat{T}_{A_{0}}(l)(\varphi) d l\right) \\
& =\int_{0}^{t} x^{*}\left(\widehat{T}_{A_{0}}(l)(\varphi)\right) d l \\
& =\int_{0}^{t} \int_{0}^{+\infty} \psi(a) \widehat{T}_{A_{0}}(l)(\varphi)(a) d a d l \\
& =\int_{0}^{t} \int_{l}^{+\infty} \psi(a) \varphi(a-l) d a d l \\
& =\int_{0}^{t} \int_{0}^{a} \psi(a) \varphi(a-l) d l d a+\int_{t}^{\infty} \int_{0}^{t} \psi(a) \varphi(a-l) d l d a \\
& =\int_{0}^{\infty} \int_{0}^{\min (a, t)} \psi(a) \varphi(a-l) d l d a \\
& =\int_{0}^{\infty} \psi(a) \int_{0}^{t} H(a-l) \varphi(a-l) d l d a .
\end{aligned}
$$

Since $x^{*}$ is arbitrary, by using the Hahn-Banach theorem we deduce that

$$
\widehat{S}_{A}(t)(0, \varphi)(a)=\int_{0}^{t} \widehat{T}_{A_{0}}(l)(\varphi)(a) d l, \forall t \geq 0, \text { for a.e. } a \geq 0
$$


and the result follows by using (5.5).

Hence using (5.6) and (5.8) we have for each $\left(\begin{array}{c}0_{\mathbb{R}^{n}} \\ \varphi\end{array}\right) \in \mathcal{C}_{0}$

$$
T_{A_{0}}(h)\left(\begin{array}{c}
0_{\mathbb{R}^{n}} \\
\varphi
\end{array}\right)+S_{A}(h) F\left(\begin{array}{c}
0_{\mathbb{R}^{n}} \\
\varphi
\end{array}\right)=\left(\begin{array}{c}
0_{\mathbb{R}^{n}} \\
\widehat{v}(\varphi ; h)
\end{array}\right), \forall h \geq 0
$$

with

$$
\widehat{v}(\varphi ; h)=\widehat{T}_{A_{0}}(h)(\varphi)+\widehat{S}_{A}(h)\left(F_{0}(\varphi), F_{1}(\varphi)\right), \forall h \geq 0 .
$$

More precisely by using (5.6) and (5.9) we have

$\widehat{v}(\varphi ; h)(a)=H(a-h) \varphi(a-h)+[1-H(a-h)] F_{0}(\varphi)+\int_{0}^{h} H(a-l) F_{1}(\varphi)(a-l) d l, \forall h \geq 0, \forall a \geq 0$

or equivalently

$$
\widehat{v}(\varphi ; h)(a)=\widehat{v}_{1}(\varphi ; h)(a)+\widehat{v}_{2}(\varphi ; h)(a), \forall h \geq 0, \forall a \geq 0
$$

with

$$
\left\{\begin{aligned}
\widehat{v}_{1}(\varphi ; h)(a)= & H(a-h) \varphi(a-h)+[1-H(a-h)] F_{0}(\varphi) \\
& +h H(a-h) F_{1}(\varphi)(a-h) \\
\widehat{v}_{2}(\varphi ; h)(a)= & h\left[F_{1}(\varphi)(a)-H(a-h) F_{1}(\varphi)(a-h)\right] \\
& +\int_{0}^{h}\left[H(a-l) F_{1}(\varphi)(a-l)-F_{1}(\varphi)(a)\right] d l .
\end{aligned}\right.
$$

Lemma 5.2 For each $\varphi \in C$ we have

$$
\lim _{h \rightarrow 0^{+}} \frac{1}{h}\left\|\widehat{v}_{2}(\varphi ; h)\right\|_{L^{p}}=0 .
$$

Proof. We will give the proof for $1<p<+\infty$. The case $p=1$ can be obtained easily. Let $q \in(1,+\infty)$ be given such that $\frac{1}{p}+\frac{1}{q}=1$. We have for each $h>0$

$$
\begin{aligned}
\left\|\widehat{v}_{2}(\varphi ; h)\right\|_{L^{p}} \leq & h\left\|H(.) F_{1}(\varphi)(.)-H(.-h) F_{1}(\varphi)(.-h)\right\|_{L^{p}} \\
& +\left(\int_{0}^{+\infty}\left(\int_{0}^{h}\left\|H(a-l) F_{1}(\varphi)(a-l)-F_{1}(\varphi)(a)\right\| d l\right)^{p} d a\right)^{\frac{1}{p}} \\
\leq & h\left\|H(.) F_{1}(\varphi)(.)-H(.-h) F_{1}(\varphi)(.-h)\right\|_{L^{p}} \\
& +h^{\frac{1}{q}}\left(\int_{0}^{+\infty} \int_{0}^{h}\left\|H(a-l) F_{1}(\varphi)(a-l)-F_{1}(\varphi)(a)\right\|^{p} d l d a\right)^{\frac{1}{p}} \\
\leq & h\left\|H(.) F_{1}(\varphi)(.)-H(.-h) F_{1}(\varphi)(.-h)\right\|_{L^{p}} \\
& +h^{\frac{1}{q}}\left(\int_{0}^{h}\left\|H(.-l) F_{1}(\varphi)(.-l)-H(.) F_{1}(\varphi)(.)\right\|_{L^{p}}^{p} d l\right)^{\frac{1}{p}} \\
\leq & h\left\|H(.) F_{1}(\varphi)(.)-H(.-h) F_{1}(\varphi)(.-h)\right\|_{L^{p}} \\
& +h\left(\int_{0}^{1}\left\|H(.-l h) F_{1}(\varphi)(.-l h)-H(.) F_{1}(\varphi)(.)\right\|_{L^{p}}^{p} d l\right)^{\frac{1}{p}}
\end{aligned}
$$


and the result follows by using the continuity of the translation in $L^{p}$.

Note that since the $d\left(. ; \mathcal{C}_{0}\right)$ is 1-Lipschitz continuous we have

$0 \leq \frac{1}{h} d\left(\left(\begin{array}{c}0_{\mathbb{R}^{n}} \\ \widehat{v}(\varphi ; h)\end{array}\right) ; \mathcal{C}_{0}\right) \leq \frac{1}{h} d\left(\left(\begin{array}{c}0_{\mathbb{R}^{n}} \\ \widehat{v}_{1}(\varphi ; h)\end{array}\right) ; \mathcal{C}_{0}\right)+\frac{1}{h}\left\|\widehat{v}_{2}(\varphi ; h)\right\|_{L^{p}}, \forall h>0$

it now follows from Lemma 5.2 that Assumption 4.4 is satisfied if

$$
\lim _{h \rightarrow 0^{+}} \frac{1}{h} d\left(\left(\begin{array}{c}
0 \mathbb{R}^{n} \\
\widehat{v}_{1}(\varphi ; h)
\end{array}\right) ; \mathcal{C}_{0}\right)=0 .
$$

In order to prove (5.13) we will show that under some conditions to be make precise later $\left(\begin{array}{c}0_{\mathbb{R}^{n}} \\ \widehat{v}_{1}(\varphi ; h)\end{array}\right)$ belongs to $\mathcal{C}_{0}$ for $h>0$ sufficiently small. To this end note that

$$
\widehat{v}_{1}(\varphi ; h)(a)=\left\{\begin{array}{lll}
\varphi(a-h)+h F_{1}(\varphi)(a-h) & \text { if } & a \geq h \\
F_{0}(\varphi) & \text { if } & a<h
\end{array}\right.
$$

Lemma 5.3 Assume that

$$
\int_{0}^{a_{\dagger}} \beta(a) d a \leq \frac{4}{\kappa}
$$

Then there exists $h_{0}>0$ such that for each $\varphi \in C$ we have

$$
\widehat{v}_{1}(\varphi ; h) \in C, \forall h \in\left(0, h_{0}\right)
$$

Proof. Let $\varphi \in C$ be given. Since $\Theta$ is linear, if $a \geq h$ then by (5.14) we have

$$
\begin{aligned}
\Theta\left(\widehat{v}_{1}(\varphi ; h)(a)\right) & =\Theta(\varphi(a-h)-h \mu(a-h) \varphi(a-h)[\kappa-\Theta(\varphi(a-h))]) \\
& =(1-h \mu(a-h)[\kappa-\Theta(\varphi(a-h)]) \Theta(\varphi(a-h))
\end{aligned}
$$

hence

$$
\left[1-h\|\mu\|_{\infty}(\kappa-\Theta(\varphi(a-h))] \Theta(\varphi(a-h)) \leq \Theta\left(\widehat{v}_{1}(\varphi ; h)(a)\right) \leq\left[1-h \mu_{-}(\kappa-\Theta(\varphi(a-h))] \Theta(\varphi(a-h))\right.\right.
$$

and since the map $s \in[0, \kappa] \rightarrow\left[1-h \mu_{-}(\kappa-s)\right] s$ is non decreasing for $h>0$ sufficiently small it follows that there exists $h_{0}>0$ depending only on $\kappa$ and $\mu$ such that

$$
0 \leq \Theta\left(\widehat{v}_{1}(\varphi ; h)(a)\right) \leq \kappa, \forall a \geq h, \forall h \in\left[0, h_{0}\right] .
$$

For $0 \leq a<h$ by using (5.14) we have

$$
\begin{aligned}
\Theta\left(\widehat{v}_{1}(\varphi ; h)(a)\right) & =\Theta\left(F_{0}(\varphi)\right) \\
& =\Theta\left(\int_{0}^{+\infty} \beta(a) \varphi(a)[\kappa-\Theta(\varphi(a))] d a\right) \\
& =\int_{0}^{a_{\dagger}} \beta(a)[\kappa-\Theta(\varphi(a))] \Theta(\varphi(a)) d a .
\end{aligned}
$$


Since the maximum of the map $s \in[0, \kappa] \rightarrow(\kappa-s) s$ is $\frac{\kappa^{2}}{4}$ we obtain that

$$
0 \leq \Theta\left(\widehat{v}_{1}(\varphi ; h)(a)\right) \leq \frac{\kappa^{2}}{4} \int_{0}^{a_{\dagger}} \beta(a) d a, \text { if } 0 \leq a<h, h>0
$$

Thus we see from (5.15) and (5.17) that

$$
0 \leq \Theta\left(\widehat{v}_{1}(\varphi ; h)(a)\right) \leq \kappa, \text { if } 0 \leq a<h, h>0 .
$$

The result follows from (5.16) and (5.18).

We have the following result.

Theorem 5.4 Assume that

$$
\int_{0}^{a_{\dagger}} \beta(a) d a \leq \frac{4}{\kappa}
$$

Then for each $u_{0} \in L_{+}^{p}\left(\mathbb{R}_{+}, \mathbb{R}^{n}\right)$ with

$$
0 \leq \Theta\left(u_{0}(a)\right) \leq \kappa, \text { for a.e. } a \in \mathbb{R}_{+}
$$

there exists a unique continuous globally defined mild solution $t \in \mathbb{R}_{+} \rightarrow u(t,.) \in$ $L_{+}^{p}\left(\mathbb{R}_{+}, \mathbb{R}^{n}\right)$ of (5.1) such that

$$
0 \leq \Theta(u(t, a)) \leq \kappa, \text { for a.e. } a \in \mathbb{R}_{+}, \forall t \geq 0 .
$$

Proof. The existence of a maximally defined solution of (5.3) satisfying (5.19) is direct application of Theorem 4.6. To obtain the global existence of the solution of (5.3) it is enough to observe that

$F\left(\begin{array}{c}0_{\mathbb{R}^{n}} \\ \varphi\end{array}\right) \leq\left(\begin{array}{c}\int_{0}^{+\infty} \beta(a) \kappa \varphi(a) d a \\ \mu(.) \kappa \varphi(.)\end{array}\right), \forall\left(\begin{array}{c}0_{\mathbb{R}^{n}} \\ \varphi\end{array}\right) \in X_{0+}:=\left\{0_{\mathbb{R}^{n}}\right\} \times L_{+}^{p}\left(\mathbb{R}_{+}, \mathbb{R}^{n}\right)$.

and infer from [9, Corollary 3.7]. The result follows by using the fact that system (5.1) coincides with (5.3) in $C$.

\section{References}

[1] W. Arendt, Resolvent positive operators, Proc. London Math. Soc. 54 (1987), 321-349.

[2] W. Arendt, Vector valued Laplace transforms and Cauchy problems, Israel J. Math. 59 (1987), 327-352.

[3] W. Arendt, C. J. K. Batty, M. Hieber, and F. Neubrander, Vector-Valued Laplace Transforms and Cauchy Problems, Birkhäuser, Basel, 2001.

[4] H. Brezis, On a characterization of flow-invariant sets, Communications on Pure and Applied Mathematics, 23(2) (1970), 261-263. 
[5] J. Dieudonne, Foundations of Modern Analysis, Academic Press Inc. 1960

[6] P. Hartman, On invariant sets and on a theorem of Wazewski, Proceedings of the American Mathematical Society, 32(2) (1972), 511-520.

[7] M. W. Hirsch and Hal Smith, Monotone dynamical systems, Handbook of differential equations: ordinary differential equations. Vol. II, (2005), 239357.

[8] P. Magal, and S. Ruan, On Integrated Semigroups and Age Structured Models in $L^{p}$ Spaces, Differential and Integral Equations, 20 (2007), 197139.

[9] P. Magal and S. Ruan, On Semilinear Cauchy Problems with Non-dense Domain, Advances in Differential Equations, 14 (2009), 1041-1084.

[10] P. Magal and S. Ruan, Theory and Applications of Abstract Semilinear Cauchy Problems Applied Mathematical Sciences, vol. 201, Springer International Publishing (2018).

[11] R. H. Martin, A Maximum Principle for Semilinear Parabolic Systems, Proc. A.M.S., 74(1) (1979), 66-70.

[12] R. Martin, H. L. Smith, Abstract functional differential equations and reaction-diffusion systems, Trans. Amer. Math. Soc., 321 (1990), 1-44.

[13] M. Nagumo, Uber die lage der integralkurven gewohnlicher differentialgleichungen, Proc. Phys. Math. Soc. Japan, 24 (1942), 551-559.

[14] N. H. Pavel and D. Motreanu, Tangency, flow invariance for differential equations, and optimization problems, vol. 219, CRC Press (1999).

[15] R. Redheffer and W. Walter, Flow-invariant sets and differential inequalities in normed spaces. Applicable Analysis, 5(2) (1975), 149-161.

[16] R. Redheffer and W. Walter, Invariant sets for systems of partial differential equations I. Parabolic equations. Archive for Rational Mechanics and Analysis, 67(1) (1977), 41-52.

[17] H. R. Thieme, Semiflows generated by Lipschitz perturbations of nondensely defined operators, Differential Integral Equations 3 (1990), 10351066.

[18] H. R. Thieme, Differentiability of convolutions, integrated semigroups of bounded semi-variation, and the inhomogeneous Cauchy problem, J. Evol. Equ. 8 (2008), 283-305.

[19] W. Walter, Ordinary differential inequalities in ordered Banach spaces. Journal of differential equations, 9(2) (1971), 253-261.

[20] G. F. Webb, Theory of Nonlinear Age-dependent Population Dynamics, Marcel Dekker, New York, 1985. 